\title{
Effects of Tai Chi training on the physical and mental health status in patients with chronic obstructive pulmonary disease: a systematic review and meta-analysis
}

\author{
Chengyao Guo ${ }^{1 \#}$, Guiling Xiang ${ }^{2 \#}$, Liang Xie ${ }^{2 \#}$, Zilong Liu ${ }^{2}$ Xiaomin Zhang ${ }^{1}$, Qinhan Wu ${ }^{2}$, Shanqun $\mathrm{Li}^{2}$, \\ Yan $W_{\mathbf{u}^{1}}$ \\ ${ }^{1}$ Nursing Department, ${ }^{2}$ Department of Pulmonary Medicine, Zhongshan Hospital, Fudan University, Shanghai 200032, China \\ Contributions: (I) Conception and design: C Guo, Y Wu, S Li; (II) Administrative support: Y Wu, S Li; (III) Provision of study materials or patients: C \\ Guo, L Xie, Z Liu, X Zhang, Q Wu, Y Wu, S Li; (IV) Collection and assembly of data: C Guo, G Xiang, L Xie; (V) Data analysis and interpretation: \\ C Guo; (VI) Manuscript writing: All authors; (VII) Final approval of manuscript: All authors. \\ \#These authors contributed equally to this work. \\ Correspondence to: Shanqun Li. Department of Pulmonary Medicine, Zhongshan Hospital, Fudan University, 180 Fenglin Rd., Shanghai 200032, \\ China. Email: 1sq18616880856@163.com; Yan Wu. Nursing Department, Zhongshan Hospital, Fudan University, 180 Fenglin Rd., Shanghai \\ 200032, China. Email: wu.yan@zs-hospital.sh.cn.
}

Background: Tai Chi is a systematic whole body movement developed in ancient China. It plays an increasingly important role in the field of pulmonary rehabilitation for patients with chronic obstructive pulmonary disease (COPD). Our review aimed to explore the impact of Tai Chi on the physical and mental health of patients with COPD.

Methods: We searched several English and Chinese databases and used the combination of subject words and free words to search for available literature from the establishment of the library until August 28, 2018. Two researchers screened studies and collected the data independently. The study inclusion criteria included: (I) patients diagnosed with COPD; (II) Tai Chi or Tai Chi Qigong as an intervention in addition to routine treatment; (III) routine treatment with or without exercises as control group. The primary outcomes were lung function, exercise capacity and health status; (IV) randomized controlled trials.

Results: Sixteen articles were included from 2009 to $2018(\mathrm{n}=1,096)$. The average time duration of Tai Chi program was 53.4 minutes each session, 4.13 sessions a week for a total of 4.13 months. Comparing with control group, Tai Chi group improved some lung function (forced volume capacity: mean difference $=0.12,95 \%$ CI: $0.03-0.21$ ), (forced expiratory volume in 1s: mean difference $=0.15,95 \%$ CI: $0.08-0.21$ ), enhanced 6-minute walking distance score (mean difference $=30.78,95 \%$ CI: 15.15-46.42), decreased COPD Assessment Test score (mean difference $=-5.00,95 \%$ CI: -7.51 to -2.50 ), decreased St. George's Respiratory Questionnaire score (mean difference $=-8.66,95 \% \mathrm{CI}:-14.60$ to -2.72 ), enhanced Chronic Respiratory Disease Questionnaire score (mean difference $=2.16$, 95\% CI: 1.49-2.83), decreased Hospital Anxiety and Depression Scale score(anxiety: mean difference $=-1.04,95 \%$ CI: -1.58 to -0.51 ; depression: mean difference $=-1.25,95 \% \mathrm{CI}:-1.77$ to -0.73 ). Comparing with exercise group, Tai Chi group statistically enhanced 6-minute walking distance score (mean difference $=7.77,95 \%$ CI: 2.63-12.91).

Conclusions: Tai Chi may represent an appropriate alternative or complement to standard rehabilitation programs. However, whether Tai Chi is better than pulmonary rehabilitation exercise has not been determined.

Keywords: Tai Chi; traditional Chinese exercise; chronic obstructive pulmonary disease (COPD); meta-analysis

Submitted Sep 05, 2019. Accepted for publication Dec 27, 2019.

doi: $10.21037 /$ jtd.2020.01.03

View this article at: http://dx.doi.org/10.21037/jtd.2020.01.03 


\section{Introduction}

Chronic obstructive pulmonary disease (COPD) is a progressive airway stenosis disease caused by chronic bronchitis or emphysema (1). According to the latest World Health Organization (WHO) statistics [2004], there are currently 64 million people with COPD and three million people died of COPD. The WHO predicts that by 2030, COPD will become the third most deadly disease in the world. In 2013, COPD is the fifth cause of reduction in Disability-Adjusted Life Year (DALY) (2). COPD imposes a huge burden on the economy and life to society and individuals. The manifestations of COPD include dyspnea, poor exercise tolerance, chronic cough with or without sputum, and wheezing to respiratory failure or pulmonary heart disease (3). These performances lead to a decrease in the patient's mobility, which seriously affects the physical and mental health and reduces the quality of life. In recent years, more and more studies have recognized the importance of pulmonary rehabilitation for patients with COPD (4). A guideline issued jointly by American College of Physicians (ACP), American College of Chest Physicians (ACCP), American Thoracic Society (ATS), and European Respiratory Society (ERS) recommended the use of pulmonary rehabilitation in patients with COPD (Grade: strong recommendation, moderate-quality evidence) (3). Exercise is the core method of pulmonary rehabilitation (4).

Tai Chi is a systematic whole body movement developed in ancient China. It includes a series of slow and rhythmic circular movements, emphasizing the use of "spirit" or attention to control breathing and body movements to promote "qi" in the body. "Qi" is considered to be a necessary substance for maintaining the body's homeostasis, it is also the root of longevity (5). Tai Chi aims to strengthen the body, improve the flow of blood and other fluids, maintain body balance, emphasize the proprioception, and feel the change of consciousness of the body moving in space (6). Due to the simplicity and zero-based nature of Tai Chi, people can practice at anytime, anywhere without any equipment. It is a worldwide exercise which is economical, convenient and suitable for all ages.

With the popularization of Tai Chi in the world, the studies about Tai Chi's improvement to patients' health outcomes have been extended to various chronic diseases. Tai Chi has become the focus of many clinical research and systematic review (6). Tai Chi plays an increasingly important role in the field of pulmonary rehabilitation for patients with COPD. In recent years, more and more clinical trials have been conducted to study the effects of Tai Chi on lung function, health status and quality of life in patients with COPD. However, the results of each study were different (7-10). In addition, there are no integrated studies on the mental impact of Tai Chi on patients with COPD. Previous systematic reviews and meta-analysis indicated that higher quality randomized controlled trials were needed in the future, as well as large sample data and longer-time intervention period interventions to support the evidence of Tai Chi in improving the health outcomes of patients with COPD $(4,11)$. Therefore, our study aimed to explore the impact of Tai Chi on the physical and mental health of patients with COPD.

\section{Methods}

This systematic review and meta-analysis was conducted in accordance with the preferred reporting items for systematic reviews and meta-analyses (PRISMA) (12). The PRISMA checklist was provided in Table S1.

We searched several English and Chinese databases: The Cochrane Library, Joanna Briggs Institute database, Embase, PubMed, Web of Science, Cumulative Index to Nursing and Allied Health Literature, China National Knowledge Infrastructure, Wanfang database, China Biology Medicine disc. We used the combination of subject words and free words to search for available literature from the establishment of the library until August 28, 2018. The search terms included: "Tai Chi", "Tai JI", "Tai Chi quan", "Tai Ji quan", and "COPD”, "chronic obstructive pulmonary disease", "chronic obstructive airway disease", "chronic obstructive lung disease". The search strategy was described in Supplementary file 1.

Our primary outcomes were lung function [such as forced volume capacity (FVC), forced expiratory volume in $1 \mathrm{~s}\left(\mathrm{FEV}_{1}\right), \mathrm{FEV}_{1}$ percent predicted normal values $\left(\mathrm{FEV}_{1} \%\right)$, the ratio of $\mathrm{FEV}_{1}$ to $\left.\mathrm{FVC}\left(\mathrm{FEV}_{1} / \mathrm{FVC}\right)\right]$, exercise capacity [such as 6-minute walking distance (6MWD)], health status [such as COPD Assessment Test (CAT)]. Secondary outcomes included the following variables: quality of life [such as St. George's Respiratory Questionnaire (SGRQ), Chronic Respiratory Disease Questionnaire (CRQ)]; mental status [such as Hospital Anxiety and Depression Scale (HAD), Self-rating Anxiety Scale (SAS) and Self-rating Depression Scale (SDS)].

The study inclusion criteria included: (I) patients diagnosed with COPD; (II) Tai Chi or Tai Chi Qigong as an intervention in addition to routine treatment; (III) routine 
treatment with or without exercises as control group. The routine treatment was medical treatment, and the exercises included respiratory exercises and physical exercises; (IV) reported at least one of the following observational parameter or outcomes: FVC, $\mathrm{FEV}_{1}, \mathrm{FEV}_{1} \%, \mathrm{FEV}_{1} / \mathrm{FVC}$, 6MWD, CAT; SGRQ, CRQ, HAD, SAS and SDS; (V) randomized controlled trials.

The study exclusion criteria included: (I) patients with cognitive or sensory impairments; (II) other chronic diseases such as neuromuscular diseases, cardiovascular diseases; (III) published languages were not Chinese or English.

The selected studies were imported into NoteExpress, and the duplicated articles were removed. Two researchers read the titles and abstracts respectively, selected the studies that met the inclusion criteria, then read the full text, and removed the studies that did not meet the inclusion criteria. Finally, 16 studies were included in our review.

The same two researchers extracted data independently according to the following information: first author, publication year, country, language of publication, sample size, age, duration of COPD, disease severity, interventions (including intervention time, frequency, grouping), outcomes, and measurement time point.

According to the Cochrane Collaboration's Risk of Bias, two researchers independently assessed the quality of the studies. The assessed items included random sequences generation, allocation concealment, blinding of participants and personnel, blinding of outcome assessment, incomplete outcome data, selective outcome reporting, and other sources of bias. Assessed criteria included low risk of bias, high risk of bias, and unclear. All inconsistent assess were resolved by a third researcher.

Statistical analysis was performed using Review Manager 5.0. The mean difference and the standard deviations of the mean difference before and after the intervention were calculated. If the outcome was measured in different ways, the standardized mean difference (SMD) and 95\% CI were used. If the outcome was measured in the same way, the mean difference (MD) and 95\% CI were used. There was a statistical difference at $\mathrm{P}<0.05$. Heterogeneity was assessed by using $\mathrm{I}^{2}$ statistic. $\mathrm{I}^{2}$ between $0-40 \%$ means that heterogeneity may not be important, $\mathrm{I}^{2}$ between $30-60 \%$ means moderate heterogeneity, and $\mathrm{I}^{2}$ between $50-90 \%$ means significant heterogeneity. $\mathrm{I}^{2}$ between $75-100 \%$ means a large heterogeneity (13). If $\mathrm{I}^{2}$ was $>50 \%$, we used the random effect model to combine the effect sizes, and if $\mathrm{I}^{2}$ was $<50 \%$, the fixed effect model was used to combine the effect sizes.

\section{Results}

The study selection flow chart was shown in Figure 1. A total of 349 related articles were retrieved, 160 duplicates were excluded, and 153 articles were excluded by reading the title and abstract. Further reading the full text, we excluded 20 articles, including three articles with inconsistent research design (14-16), two articles with inconsistent outcomes $(17,18)$, five articles without relative outcomes (19-23), five articles with different study types (24-28), four articles with inconsistent interventions $(7,9,29,30)$, and one article with unclear interventions which could not be judged (31). Finally, 16 articles were included in the systematic review $(8,10,32-45)$.

The detailed characteristics of the included studies were shown in Table 1. There are nine articles in English $(8,10,32,36,37,39-41,44)$, and seven articles in Chinese (33-35,38,42,43,45). Two Chinese articles published by Du were conducted by the same team $(42,43)$. Three English articles published by Chan were conducted by the same team $(39,41,44)$. In 13 studies, the sample size ranged from 10 to 206, with total of 1,096 samples. The intervention to intervention group was Tai Chi, four articles used simple Tai Chi $(10,33,38,45)$, four articles used Yang style $(8,32,42,43)$, two articles used Sun style $(36,40)$, three articles used Tai Chi Qigong $(39,41,44)$, three articles did not mention the style $(34,35,37)$. Among the control group, routine treatment were used in six studies $(8,10,33,37,40,45)$, exercise were adopted in three articles $(32,34,36)$. And seven articles used two control groups, one using routine treatment and the other using exercise (35,38,39,41-44). The exercises included respiratory exercises and physical exercises. Intervention time ranged from two months to 12 months. We divided intervention duration into short-time intervention period ( $<3$ months), mediumtime intervention period (4-6 months), and long-time intervention period ( $>6$ months). The frequency of intervention was two to five times per week with a duration of 30-60 minutes every time.

For primary outcomes, there were 14 articles which reported pulmonary function using $\mathrm{FVC}, \mathrm{FEV}_{1}, \mathrm{FEV}_{1} \%$, $\mathrm{FEV}_{1} / \mathrm{FVC}(8,10,32-38,41-45), 12$ articles which reported exercise capacity using 6MWD (8,10,32-34,36-38, 41-44), and five articles using CAT to assess health status $(10,33,34,38,43)$. For second outcomes, there were five articles using SGRQ to assess quality of life $(32,33,36,39,42)$, two articles using CRQ to assess quality of life $(8,40)$, two articles using HADS to assess anxiety 


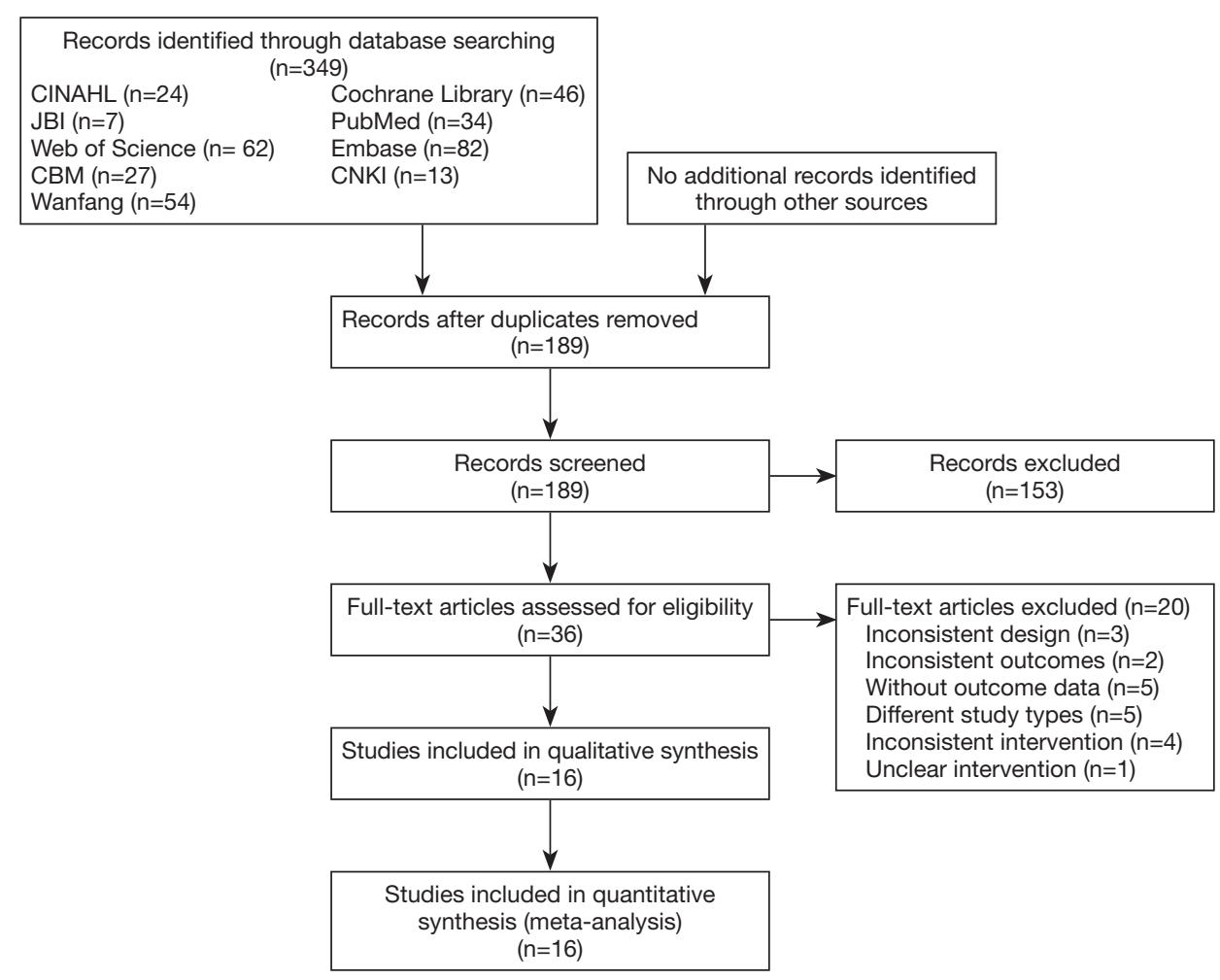

Figure 1 Flowchart of study selection.

and depression $(33,40)$, and one article using SAS and SDS to assess anxiety and depression (34).

Table 2 shows the risk of bias for included studies. Figure 2 shows methodological quality. Figure 3 shows methodological quality summary. Twelve articles described the method of random sequences generation $(8,10,33,35-42,44)$; Seven articles described allocation concealment $(10,36,37,39-$ $41,44)$; only five articles mentioned the implementation of blinding of participants and personnel $(8,10,37,39,40,45)$; seven articles mentioned the implementation of blinding of outcome assessment (8,36,37,39-41,45); 14 articles conducted an intention-to-treat analysis to the patient's loss of followup (8,10,32-38,40,42-45); All included articles reported all relative outcomes; baseline was inconsistent in one article (10).

There were 16 articles which had available data for meta-analysis. The forest plots showed the results of meta-analysis (Figures 4-13). Table 3 summarized the results of meta-analysis. Comparing with control group, Tai Chi group improved some lung function, enhanced 6MWD score, decreased CAT score, decreased SGRQ score, enhanced CRQ score, decreased HAD score. Comparing with exercise group, Tai Chi group statistically enhanced 6MWD.

\section{Discussion}

GOLD classifies COPD as "a disease state characterized by airflow limitation and incompletely reversible" (46). It would be difficult for most patients to recover to normal lung function after being diagnosed with COPD. The metaanalysis of lung function showed that compared with the control group, the FVC, $\mathrm{FEV}_{1}$ were significantly enhanced in the Tai Chi group. Compared with the exercise group, the $\mathrm{FEV}_{1} / \mathrm{FVC}$ in the Tai Chi group were significantly enhanced in the short time intervention period. Tai Chi can alleviate the symptoms of dyspnea and relieve the decline of lung function (4). However, intervention duration in the included studies for assessing lung function was mostly short-time intervention period and medium-time intervention period. Therefore, more Tai Chi studies with long-time intervention period are needed.

The 6 MWD plays a key role in assessing functional exercise capacity, estimating prognosis, and assessing treatment response in patients with COPD (47). However, the results of assessing the effects of Tai Chi on the exercise 


\begin{tabular}{|c|c|c|c|c|c|c|c|c|c|c|c|c|}
\hline Article, year & $\begin{array}{l}\text { Country of } \\
\text { performed }\end{array}$ & $\begin{array}{l}\text { Language of } \\
\text { publication }\end{array}$ & $\begin{array}{l}\text { Sample } \\
\text { size, N }\end{array}$ & $\begin{array}{l}\text { dropout in each group with } \\
\text { reasons [number] }\end{array}$ & $\begin{array}{l}\text { Age, y, mean } \\
\text { [SD/range] }\end{array}$ & $\begin{array}{l}\text { Duration of } \\
\text { COPD, y }\end{array}$ & Disease severity & Comparison arms & Treatment dosage & Exercise contents & Outcomes & Time point \\
\hline $\begin{array}{l}\text { Zhu et al. } \\
2018(10)\end{array}$ & China & English & $\begin{array}{l}\text { G1: } 30 ; \\
\text { G2: } 30\end{array}$ & $\begin{array}{l}\text { G1: had no interest [3]; } \\
\text { outside Changsha [2]; } \\
\text { G2: Refused [2]; } \\
\text { outside Changsha [1] }\end{array}$ & $\begin{array}{l}\text { G1: } 67.87 \\
{[5.22] . \mathrm{G} 2:} \\
68.10[6.57]\end{array}$ & $\begin{array}{l}\text { G1: } 7.3[4.8] . \\
\text { G2: } 8.2[6.4]\end{array}$ & $\begin{array}{l}\text { G2: 22\%; G3: } \\
\text { 42\%; G4: 36\%. } \\
\text { Total mean FEV } \\
\text { 37.94 [14.85] }\end{array}$ & $\begin{array}{l}\text { G1: TC (simplified } \\
\text { and modified } \\
\text { 24-form Tai Chi); } \\
\text { G2: control }\end{array}$ & $\begin{array}{l}40-50 \mathrm{~min} / \mathrm{session} ; 3 \\
\text { sessions/week; } 3 \text { months }\end{array}$ & NA & $\begin{array}{l}\left.\text { (I) Lung function (FEV }{ }_{1} \%\right) ; \\
\text { (II) Exercise capacity (6MWD); } \\
\text { (III) Health status (CAT) }\end{array}$ & $\begin{array}{l}3 \text { months; } \\
9 \text { months }\end{array}$ \\
\hline $\begin{array}{l}\text { Pan et al. } \\
2018(33)\end{array}$ & China & Chinese & $\begin{array}{l}\text { G1: 23; } \\
\text { G2: } 23\end{array}$ & $\begin{array}{l}\text { G1: other disease [1]; } \\
\text { refused [2]; } \\
\text { G2: refused [1]; } \\
\text { outside Sichuan [1] }\end{array}$ & NA & NA & $\begin{array}{l}\text { G1: } \mathrm{FEV}_{1} \% 52.80 \\
\text { [3.12]; G2: } \mathrm{FEV}_{1} \% \\
52.05[3.27]\end{array}$ & $\begin{array}{l}\text { G1: TC (simplified } \\
\text { and modified 24-form } \\
\text { Tai Chi). } \\
\text { G2: control }\end{array}$ & $\begin{array}{l}30 \mathrm{~min} / \mathrm{session} ; 3 \text { sessions/ } \\
\text { week; } 2 \text { months }\end{array}$ & NA & $\begin{array}{l}\text { (I) HRQoL (SGRQ); (II) Exercise capacity } \\
\text { (6MWD); (III) Lung function (FVC, FEV }, \\
\text { FVC\%, FEV } \\
\text { (HAD); ; (M) Health status (CAT) }\end{array}$ & 2 months \\
\hline $\begin{array}{l}\text { Polkey } 2018 \\
\text { (32) }\end{array}$ & China & English & $\begin{array}{l}\text { G1: } 60 \\
\text { G2: } 60\end{array}$ & $\begin{array}{l}\text { G1: adverse event [2]; } \\
\text { other reasons [3]. } \\
\text { G2: adverse event [2]; } \\
\text { other reasons [3] }\end{array}$ & NA & NA & $\begin{array}{l}\text { Total mean } \\
\text { FEV1\% } \\
\text { predicted: } 43.6\end{array}$ & $\begin{array}{l}\text { G1: TC (24 form Yang } \\
\text { style); G2: exercise }\end{array}$ & $\begin{array}{l}\text { G1: } 1 \mathrm{~h} / \text { session; } 5 \text { sessions/ } \\
\text { week; } 3 \text { months. G2: } 1 \mathrm{~h} / \\
\text { session; } 3 \text { sessions/week; } 3 \\
\text { months }\end{array}$ & $\begin{array}{l}50 \% \text { resistance exercises (arm } \\
\text { and leg weights aiming for a target } \\
70-80 \% \text { of their one-repetition } \\
\text { maximum), hybrid (rowing machine), } \\
\text { and } 50 \% \text { progressive aerobic whole } \\
\text { body exercise (e.g., cycle or treadmill) }\end{array}$ & $\begin{array}{l}\text { (I) Exercise capacity (6MWT); (II) Lung } \\
\text { function (FEV }{ }_{1}, \mathrm{FVC} \text { ); (III) HRQoL (SGRQ) }\end{array}$ & $\begin{array}{l}3 \text { months; } \\
6 \text { months }\end{array}$ \\
\hline Ni 2017 (35) & China & Chinese & $\begin{array}{l}\text { G1: } 30 ; \\
\text { G2: } 30 ; \\
\text { G3: } 30\end{array}$ & NA & $\begin{array}{l}\text { G1: } 65.3[2.1] ; \\
\text { G2: } 63.3[2.2] ; \\
\text { G3: } 60.6[4.5]\end{array}$ & $\begin{array}{l}\text { G1: } 10.9 \text { [2.2]; } \\
\text { G2: } 11.6[2.5] ; \\
\text { G3: } 9.6[3.5]\end{array}$ & NA & $\begin{array}{l}\text { G1: TC; G2: Yoga; } \\
\text { G3: control }\end{array}$ & $\begin{array}{l}\text { G1: } 30 \mathrm{~min} / \text { day; } 6 \text { months. } \\
\text { G2: } 10 \mathrm{~min} / \mathrm{session} ; 3 \\
\text { sessions/day; } 6 \text { months }\end{array}$ & NA & Lung function (FVC, $\left.\mathrm{FEV}_{1}, \mathrm{FEV}_{1} \%\right)$ & 6 months \\
\hline $\begin{array}{l}\text { Ren et al. } \\
2017 \text { (34) }\end{array}$ & China & Chinese & $\begin{array}{l}\text { G1: } 30 ; \\
\text { G1: } 30\end{array}$ & NA & $\begin{array}{l}\text { G1: } 59.2[4.5] ; \\
\text { G2: } 58.9[4.2]\end{array}$ & $\begin{array}{l}\text { G1: } 2.4[1.9] ; \\
\text { G2: } 2.3[1.8]\end{array}$ & $\begin{array}{l}\text { Total: G2: 30; } \\
\text { G3: } 30\end{array}$ & $\begin{array}{l}\text { G1: TC; } \\
\text { G2: exercise } \\
\text { (BE + PE) }\end{array}$ & $\begin{array}{l}1 \mathrm{~h} / \text { session; } 2 \text { sessions/ } \\
\text { week; } 3 \text { months }\end{array}$ & $\begin{array}{l}\text { Reducing lip breathing, abdominal } \\
\text { breathing exercise, breathing muscle } \\
\text { strength exercise, power car exercise, } \\
\text { plate exercise }\end{array}$ & $\begin{array}{l}\text { (I) Exercise capacity (6MWT); } \\
\left.\text { (II) Lung function (FEV } 1 / F V C, F V C, F E V_{1}\right) \text {; } \\
\text { (III) Health status (CAT); } \\
\text { (IV) Anxiety and Depression [SAS,SDS] }\end{array}$ & 3 months \\
\hline $\begin{array}{l}\text { Ng et al. } \\
2014 \text { (36) }\end{array}$ & $\begin{array}{l}\text { Hong Kong, } \\
\text { China }\end{array}$ & English & $\begin{array}{l}\text { G1: 94; } \\
\text { G2: 98 }\end{array}$ & $\begin{array}{l}\text { G1, G2: health problem; } \\
\text { hospital admission; } \\
\text { no interest; need family members to } \\
\text { take them to attend lessons; refused }\end{array}$ & $\begin{array}{l}\text { G1: } 74.16 \\
\text { [6.46]; G2: } \\
74.13[6.81]\end{array}$ & $\begin{array}{l}\text { G1: 7.11 } \\
\text { [10.33]; G2: } \\
\text { 8.68 [10.99] }\end{array}$ & $\begin{array}{l}\text { Total: G1 [\%]: } 39 \\
\text { [20.3]; G2 [\%]: } 78 \\
\text { [40.0]; G3 [\%]: } 58 \\
\text { [30.2]; G4 [\%]: } 17 \\
\text { [8.9] }\end{array}$ & $\begin{array}{l}\text { G1: TC (Sun-style } \\
\text { TC); G2: exercise }\end{array}$ & $\begin{array}{l}80 \mathrm{~min} / \text { session; } \\
5-7 \text { sessions/week; } 6 \\
\text { months }\end{array}$ & $\begin{array}{l}5 \text { min warm-up exercises, two } \\
\text { aerobic activities including treadmill } \\
\text { exercise and lower limb ergometry } \\
\text { exercise lasting for } 20 \text { min }\end{array}$ & $\begin{array}{l}\text { (I) HRQoL (SGRQ-HKC); } \\
\text { (II) Exercise capacity (6MWT); } \\
\text { (III) Lung function (FEV,/FVC, FEV, FVC) }\end{array}$ & $\begin{array}{l}2 \text { months; } \\
6 \text { months }\end{array}$ \\
\hline $\begin{array}{l}\text { Zhang et al. } \\
2014 \text { (38) }\end{array}$ & China & Chinese & $\begin{array}{l}\text { G1: 18; } \\
\text { G2: 18; } \\
\text { G3: 18; } \\
\text { G4: } 18\end{array}$ & NA & $\begin{array}{l}\text { G1: } 68.02 \\
\text { [6.91]; G2: } \\
\text { 67.21 [5.96]; } \\
\text { G3: 66.82 } \\
\text { [6.33]; G4: } \\
\text { 66.71 [5.84] }\end{array}$ & $\begin{array}{l}\text { G1: } 33.41 \\
\text { [2.45]; G2: } \\
\text { 32.92 [2.15]; } \\
\text { G3: } 35.32 \\
\text { [2.35]; G4: } \\
\text { 32.84 [1.98] }\end{array}$ & $\begin{array}{l}\text { G1: 11; G2: 54; } \\
\text { G3: } 7\end{array}$ & $\begin{array}{l}\text { G1: TC (simplified } \\
\text { and modified 24-form } \\
\text { Tai Chi); G2: Exercise } \\
\text { (BE); G3: TC + } \\
\text { exercise; G4: control }\end{array}$ & $\begin{array}{l}\text { G1: } 1 \text { h/day; } \\
12 \text { months. } \\
\text { G2: } 45 \text { min/day; } \\
12 \text { moths }\end{array}$ & $\begin{array}{l}\text { Combined constricted abdominal } \\
\text { breathing }\end{array}$ & $\begin{array}{l}\text { (I) Lung function (FEV }, \text { FEV } / \text { FVC, FEV } \% \text { \%); (II) } \\
\text { Exercise capacity ( }(\mathrm{MWWT}) \text {; } \\
\text { (III) Health status (CAT) }\end{array}$ & 12 months \\
\hline $\begin{array}{l}\text { Niu et al. } \\
2014(37)\end{array}$ & China & English & $\begin{array}{l}\text { G1: 20; } \\
\text { G2: } 20\end{array}$ & G2: Died [1] & $\begin{array}{l}\text { G1: } 59.7[2.76] ; \\
\text { G2: } 61.3[2.89]\end{array}$ & NA & 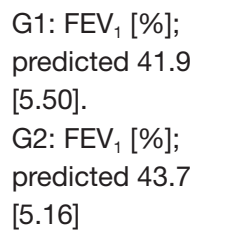 & G1: TC; G2: control & $\begin{array}{l}1 \text { session/day; } 4 \text { sessions } \\
\text { of supervised TC and } 3 \\
\text { sessions of home-based } \\
\text { TC/week; } 6 \text { months }\end{array}$ & NA & $\begin{array}{l}\text { (I) Lung function (FEV }, \mathrm{FEV}_{1} \% \text { predicted); } \\
\text { (II) Exercise capacity (6MWD) }\end{array}$ & 6 months \\
\hline $\begin{array}{l}\text { Chan } 2013 \\
\text { (39) }\end{array}$ & $\begin{array}{l}\text { Hong Kong, } \\
\text { China }\end{array}$ & English & $\begin{array}{l}\text { G1: } 70 ; \\
\text { G2: } 69 ; \\
\text { G3: } 67\end{array}$ & $\begin{array}{l}\text { G1: joint pain [3]; hospital admission [4]; } \\
\text { no interest [3]; Increased SOB [3]; Died [2]; } \\
\text { not in Hong Kong [5]; no time [1]. G2: joint } \\
\text { pain [2]; hospital admission [3]; no interest } \\
\text { [10]; Increased SOB [2]; Died [1]; refused } \\
\text { [2]; far from home [1]; default [2]. G3: no } \\
\text { interest [27]; hospital admission [3]; default } \\
\text { [3]; increased SOB [2] }\end{array}$ & $\begin{array}{l}\text { G1: } 71.7[8.2] ; \\
\text { G2: } 73.6[7.5] ; \\
\text { G3: } 73.6[7.4]\end{array}$ & $\begin{array}{l}\text { G1: } 10.3[9.3] ; \\
\text { G2: } 10.6[8.8] ; \\
\text { G3: } 12.4[10.6]\end{array}$ & $\begin{array}{l}\text { G1 [\%]: } 32[15.5] ; \\
\text { G2 [\%]: } 86 \text { [41.7]; } \\
\text { G3 [\%]: } 88 \text { [42.8] }\end{array}$ & $\begin{array}{l}\text { G1: TCQ; G2: } \\
\text { exercise (BE + PE); } \\
\text { G3: control }\end{array}$ & $\begin{array}{l}\text { G1: two 60-minute sessions/ } \\
\text { week; } 3 \text { months. G2: NA }\end{array}$ & $\begin{array}{l}\text { breathing exercise combined with } \\
\text { walking as an exercise }\end{array}$ & HRQoL (SGRQ) & $\begin{array}{l}6 \text { weeks; } \\
3 \text { months; } \\
6 \text { months }\end{array}$ \\
\hline
\end{tabular}

Table 1 (continued) 
Table 1 (continued)

\begin{tabular}{|c|c|c|c|c|c|c|c|c|c|c|c|c|}
\hline Article, year & $\begin{array}{l}\text { Country of } \\
\text { performed }\end{array}$ & $\begin{array}{l}\text { Language of } \\
\text { publication }\end{array}$ & $\begin{array}{l}\text { Sample } \\
\text { size, N }\end{array}$ & $\begin{array}{l}\text { dropout in each group with } \\
\text { reasons [number] }\end{array}$ & $\begin{array}{l}\text { Age, y, mean } \\
{[\mathrm{SD} / \text { range }]}\end{array}$ & $\begin{array}{l}\text { Duration of } \\
\text { COPD, y }\end{array}$ & Disease severity & Comparison arms & Treatment dosage & Exercise contents & Outcomes & Time point \\
\hline $\begin{array}{l}\text { Leung } 2013 \\
\text { (40) }\end{array}$ & Australia & English & $\begin{array}{l}\text { G1: 22; } \\
\text { G2: 20 }\end{array}$ & $\begin{array}{l}\text { G1: exacerbation [2]; } \\
\text { other disease [1]. } \\
\text { G2: work commitment [1] }\end{array}$ & Total: $73[8]$ & NA & $\begin{array}{l}\text { Total mean FEV, } \% \\
\text { predicted } 59[16]\end{array}$ & $\begin{array}{l}\text { G1: TC (Short-form } \\
\text { Sun-style tai chi). G2: } \\
\text { control }\end{array}$ & $\begin{array}{l}1 \mathrm{~h} / \text { session; } 2 \text { supervised } \\
\text { training sessions /week; and } \\
30 \text { min/session; } 5 \text { sessions/ } \\
\text { week; } 3 \text { months }\end{array}$ & NA & $\begin{array}{l}\text { (I) HRQoL (CRQ); } \\
\text { (II) anxiety and depression (HADS) }\end{array}$ & 3 months \\
\hline $\begin{array}{l}\text { Chan } 2013 \\
(41)\end{array}$ & $\begin{array}{l}\text { Hong Kong, } \\
\text { China }\end{array}$ & English & $\begin{array}{l}\text { G1: } 70 \\
\text { G2: } 69 \\
\text { G3: } 67\end{array}$ & $\begin{array}{l}\text { G1: Not in Hong Kong [5]; hospital } \\
\text { admission [1]; no interest [1]; increased } \\
\text { SOB [1]; no time [1]; died [1]. G2 : refused } \\
\text { [2]; default [2]. G3: no interest [16] }\end{array}$ & $\begin{array}{l}\text { G1: } 71.7[8.2] ; \\
\text { G2: } 73.6[7.5] ; \\
\text { G3: 73.6 [7.4] }\end{array}$ & $\begin{array}{l}\text { G1: } 10.3[9.3] ; \\
\text { G2: } 10.6[8.8] ; \\
\text { G3: } 12.4[10.6]\end{array}$ & $\begin{array}{l}\text { G1: FEV }{ }_{1}[\%] ; \\
\text { Predicted 50.1 } \\
\text { [21.8]. G2: FEV } \\
\text { [\%]; Predicted } \\
\text { 56.4 [25.6]. } \\
\text { G3: FEV } 1 \text { [\%]; } \\
\text { Predicted 55.1 } \\
\text { [23.3] }\end{array}$ & $\begin{array}{l}\text { G1: TCQ (13-form } \\
\text { TCQ); G2: exercise } \\
\text { (BE + PE); G3: control }\end{array}$ & $\begin{array}{l}\text { G1: } 1 \text { h/session; } 2 \text { sessions/ } \\
\text { week; } 3 \text { months. G2: NA. }\end{array}$ & $\begin{array}{l}\text { use pursed-lip breathing (PLB) and } \\
\text { diaphragmatic } \\
\text { breathing (DB) techniques }\end{array}$ & $\begin{array}{l}\text { (I) Lung function (FVC, FEV }) \text {; } \\
\text { (II) Exercise capacity (6MWD) }\end{array}$ & $\begin{array}{l}3 \text { months; } \\
6 \text { months }\end{array}$ \\
\hline $\begin{array}{l}\text { Duet al. } \\
2013(42)\end{array}$ & China & Chinese & $\begin{array}{l}\text { G1: } 36 ; \\
\text { G2: } 38 ; \\
\text { G3: } 38\end{array}$ & NA & $\begin{array}{l}\text { G1: } 65.24 \\
\text { [8.37]; G2: } \\
\text { 62.38 [6.24]; } \\
\text { G3: 64.48 } \\
\text { [6.54] }\end{array}$ & $\begin{array}{l}\text { G1: 7.18 } \\
\text { [2.72]; G2: } \\
\text { 7.82 [3.09]; } \\
\text { G3: } 7.50 \text { [2.85] }\end{array}$ & $\begin{array}{l}\text { G1: } 33 \\
\text { G2: } 79\end{array}$ & $\begin{array}{l}\text { G1: TC (24-form } \\
\text { Yang-form Tai } \\
\text { Jiquan); G2: exercise } \\
\text { (BE + PE); G3: control }\end{array}$ & $\begin{array}{l}\text { G1: } 1 \mathrm{~h} / \text { day; and } 1 \mathrm{~h} / \\
\text { session; } 2 \text { sessions/week; } \\
3 \text { months. G2: } 1.5 \mathrm{~h} / \text { day; } 3 \\
\text { months }\end{array}$ & $\begin{array}{l}\text { Narrow lip abdominal } \\
\text { breathing and walking }\end{array}$ & $\begin{array}{l}\text { (I) HRQoL (SGRQ); } \\
\text { (II) Exercise capacity (6MWD); } \\
\text { (III) Lung function [FEV } \% \text { ] }\end{array}$ & $\begin{array}{l}6 \text { weeks; } \\
3 \text { months }\end{array}$ \\
\hline $\begin{array}{l}\text { Du et al. } \\
2013(43)\end{array}$ & China & Chinese & $\begin{array}{l}\text { G1: } 36 ; \\
\text { G2: } 38 ; \\
\text { G3: } 38\end{array}$ & NA & $\begin{array}{l}\text { G1: } 65.24 \\
\text { [8.37]; G2: } \\
\text { 62.38 [6.24]; } \\
\text { G3: 64.48 } \\
\text { [6.54] }\end{array}$ & $\begin{array}{l}\text { G1: 7.18 } \\
\text { [2.72]; G2: } \\
\text { 7.82 [3.09]; } \\
\text { G3: } 7.50 \text { [2.85] }\end{array}$ & G1: 33; G2: 79 & $\begin{array}{l}\text { G1: TC (24-form } \\
\text { Yang-form Tai } \\
\text { Jiquan); G2: exercise } \\
\text { (BE + PE); G3: control }\end{array}$ & $\begin{array}{l}\text { G1: } 1 \mathrm{~h} / \text { day; and } 1 \mathrm{~h} / \\
\text { session; } 2 \text { sessions/week; } \\
3 \text { months. G2: } 1.5 \mathrm{~h} / \text { day; } 3 \\
\text { months }\end{array}$ & $\begin{array}{l}\text { Narrow lip abdominal } \\
\text { breathing and walking }\end{array}$ & $\begin{array}{l}\text { (I) Lung function (FEV } \% \text {, FEV } 1 / F V C) \text {; } \\
\text { (II) Exercise capacity (6MWD); } \\
\text { (III) Health status (CAT) }\end{array}$ & $\begin{array}{l}6 \text { weeks; } \\
3 \text { months }\end{array}$ \\
\hline $\begin{array}{l}\text { Chan } 2011 \\
(44)\end{array}$ & $\begin{array}{l}\text { Hong Kong, } \\
\text { China }\end{array}$ & English & $\begin{array}{l}\text { G1: } 70 ; \\
\text { G2: } 69 ; \\
\text { G3: } 67\end{array}$ & $\begin{array}{l}\text { G1: hospital admission [4]; no interest [2]; } \\
\text { increased SOB [2]; joint pain [3]; died [1]. } \\
\text { G2: hospital admission [3]; no interest [10]; } \\
\text { increased SOB [2]; joint pain [2]; Died [1]; } \\
\text { Venue too far from home [1]. G3: no interest } \\
\text { [11]; Increased SOB [2]; hospital admission } \\
\text { [3]; default [3] }\end{array}$ & $\begin{array}{l}\text { G1: } 71.7[8.2] ; \\
\text { G2: } 73.6[7.5] ; \\
\text { G3: } 73.6[7.4]\end{array}$ & $\begin{array}{l}\text { G1: } 10.3[9.3] ; \\
\text { G2: } 10.6[8.8] ; \\
\text { G3: } 12.4[10.6]\end{array}$ & $\begin{array}{l}\text { Total: G1 [\%]: } 32 \\
\text { [15.5]; G2 [\%]; } 86 \\
\text { [41.7]; G3 [\%]: } 88 \\
{[42.8]}\end{array}$ & $\begin{array}{l}\text { G1: TCQ (13-form } \\
\text { TCQ) G2: exercise (BE } \\
\text { + PE); G1: control }\end{array}$ & $\begin{array}{l}\text { G1: } 1 \text { hour/session; } 2 \\
\text { sessions/week; } 3 \text { months. } \\
\text { G2: } 1 \text { hour/day; } 3 \text { months }\end{array}$ & $\begin{array}{l}\text { pursed-lip breathing (PLB) and } \\
\text { diaphragmatic breathing (DB) } \\
\text { coordinated with self-paced walking }\end{array}$ & $\begin{array}{l}\text { (I) Exercise capacity (6MWD); } \\
\text { (II) Lung function (FEV }, \text { FVC) }\end{array}$ & $\begin{array}{l}6 \text { weeks; } \\
3 \text { months }\end{array}$ \\
\hline $\begin{array}{l}\text { Yeh et al. } \\
2010 \text { (8) }\end{array}$ & America & English & $\begin{array}{l}\text { G1: } 5 ; \\
\text { G2: } 5\end{array}$ & G1: discontinued intervention [1] & $\begin{array}{l}\text { G1: } 65 \text { [6]; G2: } \\
66 \text { [6] }\end{array}$ & NA & $\begin{array}{l}\text { Total mean } \mathrm{FEV}_{1} \% \\
\text { predicted } 50[7]\end{array}$ & $\begin{array}{l}\text { G1: TC (Yang-style } \\
\text { short form); G2: } \\
\text { control }\end{array}$ & $\begin{array}{l}1 \text { hour/session; } 2 \text { sessions/ } \\
\text { week; } 3 \text { months }\end{array}$ & NA & $\begin{array}{l}\text { (I) HRQoL (CRQ); (II) Exercise capacity } \\
\text { (6MWT); (III) Lung function (FEV, FVVC) }\end{array}$ & 3 months \\
\hline $\begin{array}{l}\text { Zhou et al. } \\
2009 \text { (45) }\end{array}$ & China & Chinese & $\begin{array}{l}\text { G1: 23; } \\
\text { G2: } 23\end{array}$ & NA & NA & NA & NA & $\begin{array}{l}\text { G1: TC (simplified } \\
\text { and modified 24-form } \\
\text { Tai Chi); G2: control }\end{array}$ & $40 \mathrm{~min} /$ day; 4 months & NA & Lung function (FEV $\left.1, F V C, \mathrm{FEV}_{1} \%\right)$ & 4 months \\
\hline
\end{tabular}

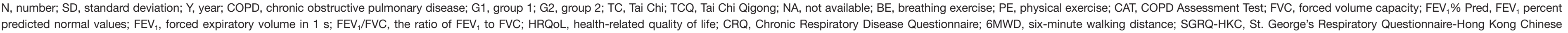
predicted normal values; FEV 1 , forced expiratory volume in $1 \mathrm{~s}$; FEV, /FVC, the ratio of FEV, to FVC; HRQoL, health-related quality of life; CRQ, Chronic Respiratory D
version; SAS, Self-rating Anxiety Scale; SDS, Self-rating Depression Scale; SGRQ, St. George's Respiratory Questionnaire; HAD, Hospital Anxiety and Depression Scale. 
Table 2 Risk of bias for included studies

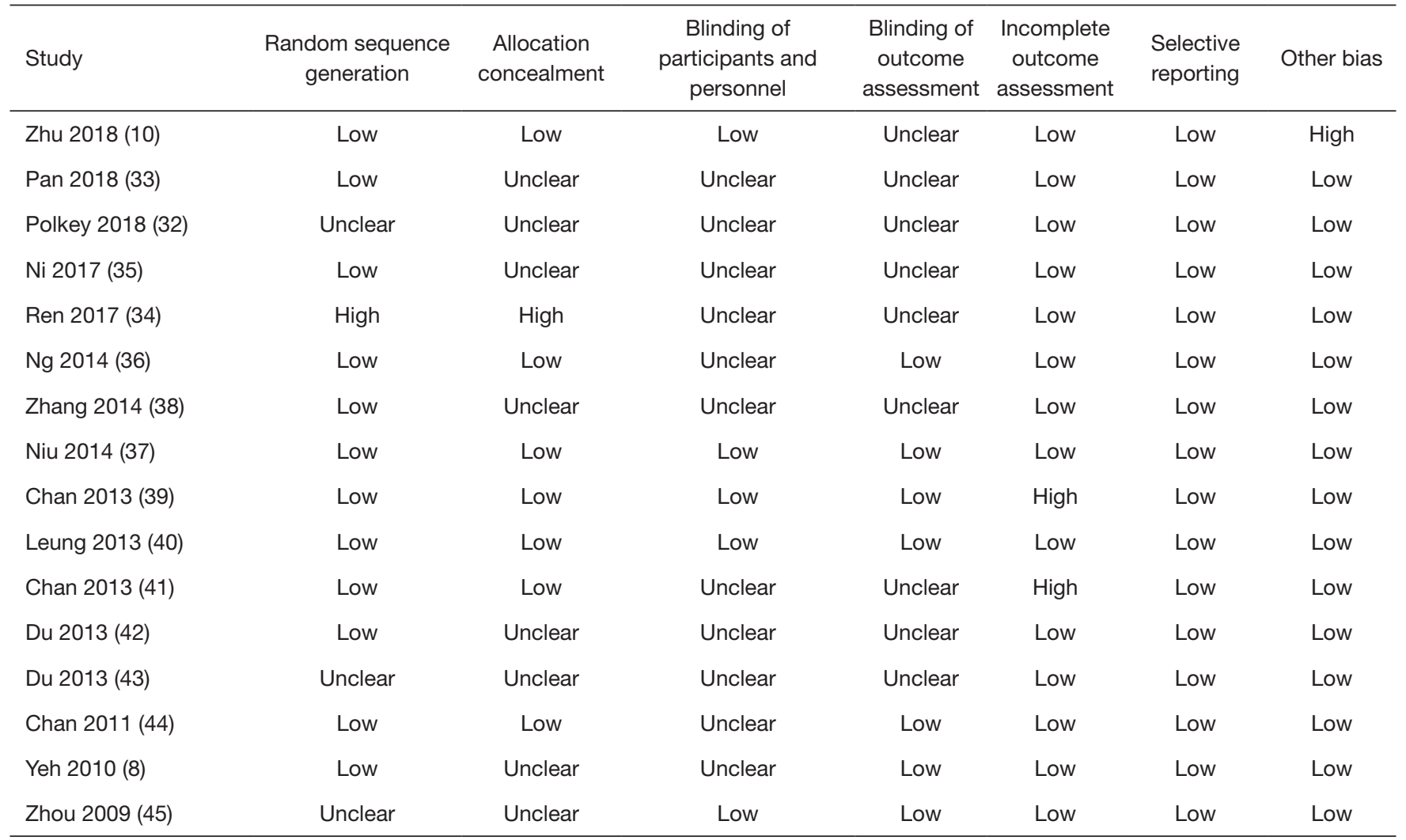

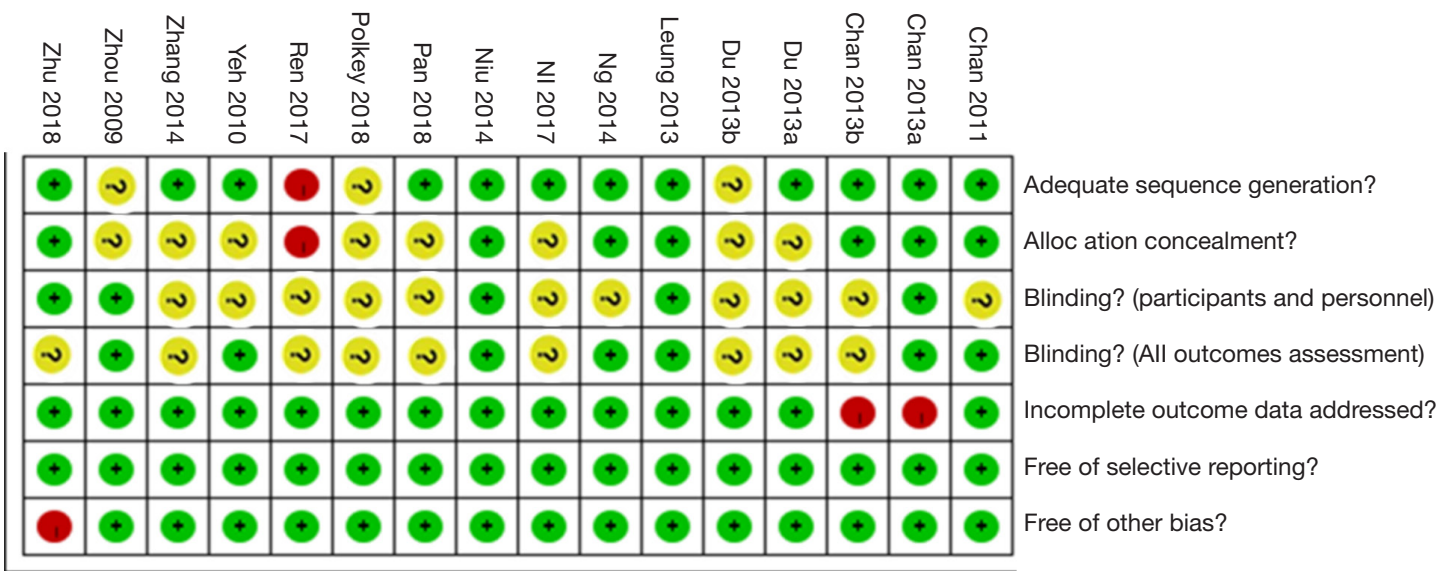

Figure 2 Methodological quality graph. 


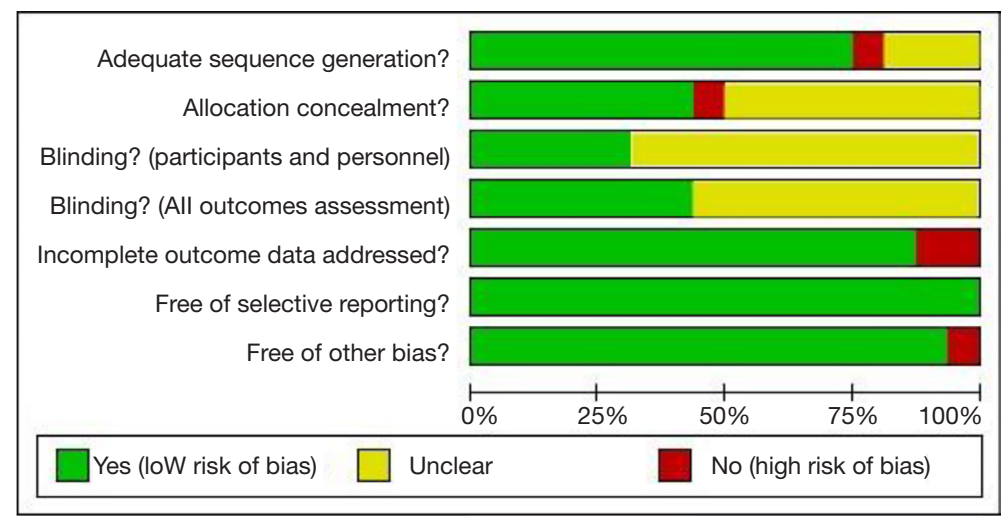

Figure 3 Methodological quality summary graph.

A

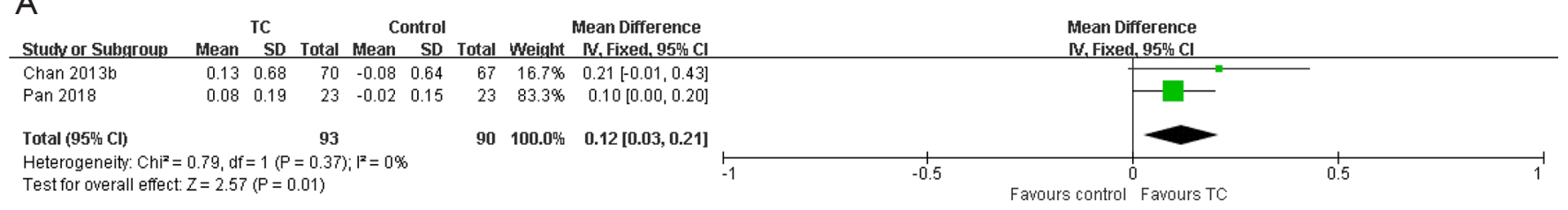

\section{B}

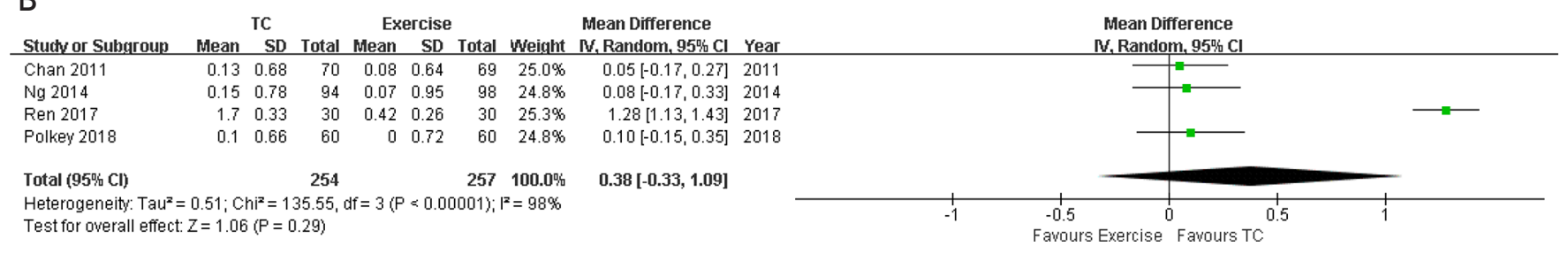

Figure 4 Meta-analysis of Tai on FVC in short time intervention period. (A) Tai Chi group versus control group; (B) Tai Chi group versus exercise group. Short time intervention period: $\leq 3$ months; medium time intervention period: $4-6$ months; long time intervention period: $>6$ months. FVC, forced volume capacity.

capacity of patients with COPD by $6 \mathrm{MWD}$ were quite different $(8,37,44)$. Pooled analysis found that compared with the control group, the $6 \mathrm{MWD}$ in the Tai Chi group was significantly improved. The difference was more obvious when the intervention time was longer. In the original study, it was found that the increase of 6MWD in the Tai Chi group was greater than the minimal clinically important differences (MCIDs) (25-35 m) recommended by patients with COPD (47). And with the extension of the intervention time, the increase of 6WMD is significantly $(10,38)$. The meta-analysis of Tai Chi group and exercise group showed that the results of 6MWD which changed by different intervention times were not consistent, and the results of the short-time intervention period intervention were significantly different, although far below the MCID.
While the results of the medium-time intervention period interventions were not statistically different. In the original study, we found that the quality of two studies in the medium-time intervention period intervention were different. The higher quality study had significant difference between the Tai Chi group and the exercise group in 6MWD (36). While in the lower quality study, only the original measurements of Tai Chi and exercise group at $6 \mathrm{WMD}$ are shown. It did not compare the $6 \mathrm{MWD}$ between the Tai Chi group and the exercise group (38). Therefore, whether there are difference in the enhance of exercise capacity between Tai Chi group and exercise group is not clear. More long-time intervention period studies are needed.

The CAT is a health assessment tool for assessing 


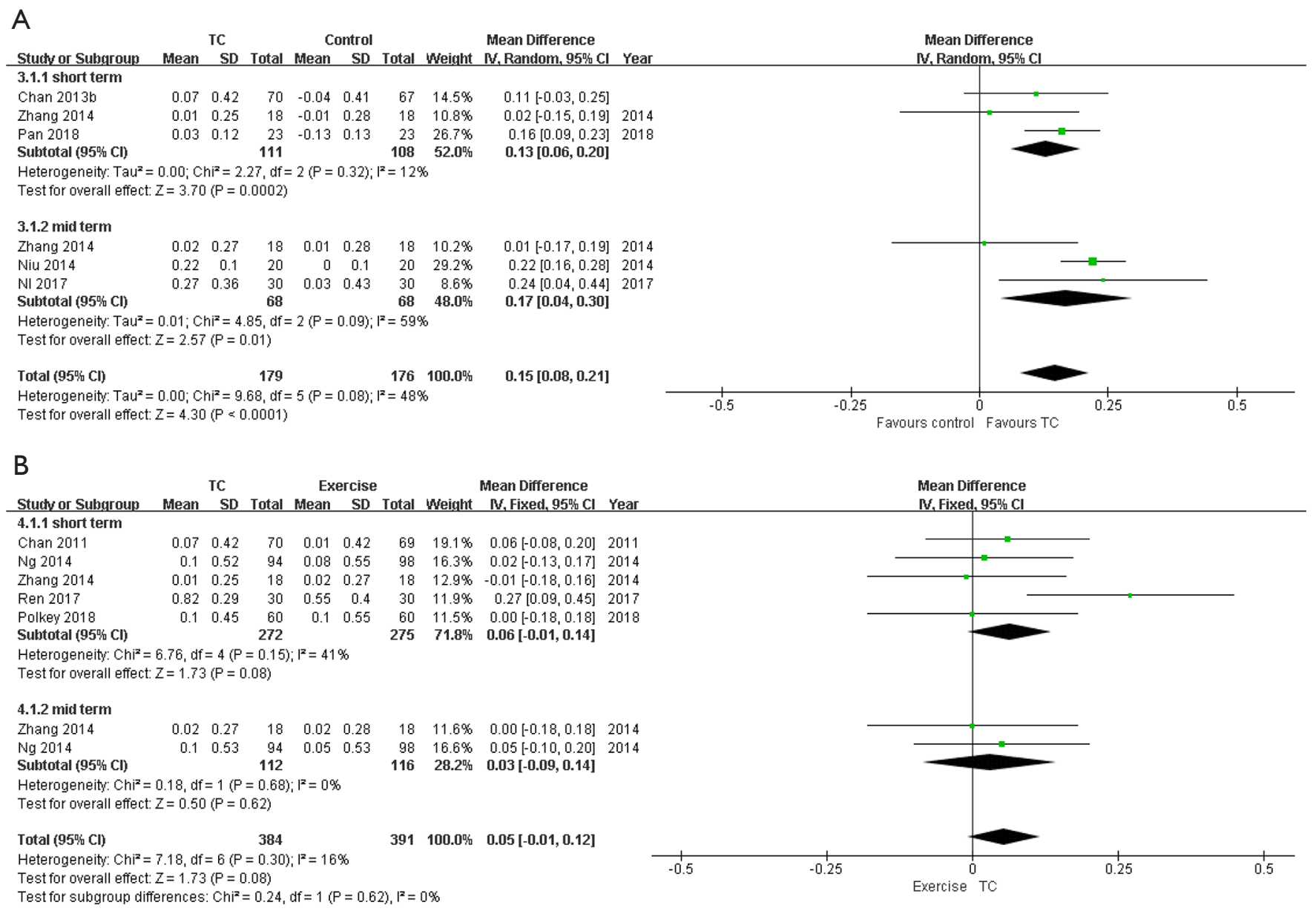

Figure 5 Meta-analysis of Tai on FEV1. (A) Tai Chi group versus control group; (B) Tai Chi group versus exercise group. Short time intervention period: $\leq 3$ months; medium time intervention period: 4-6 month; long time intervention period: $>6$ months. FEV1, forced expiratory volume in $1 \mathrm{~s}$.

the disease status of patients with COPD. It can assess the degree of lung function recovery in patients with COPD (48). The pooled analysis showed that there was a significant difference about CAT scores between the Tai Chi group and the control group in all intervention time. Moreover, the major of CAT scores in the Tai Chi group in each study were greater than the MCID (2 points) (48). It indicated that Tai Chi could significantly improve the health status of patients with COPD. There is no significant difference in the CAT scores between the Tai Chi group and the exercise group. The reason may be that Tai Chi, breathing exercise and walking exercise can enhance the respiratory muscle strength of patients with COPD, help inhale oxygen, excrete carbon dioxide, and relieve symptoms of dyspnea. However, the intervention times were all short- time intervention period in included studies comparing Tai Chi with exercise. Therefore, the effect of Tai Chi and exercise on the improvement of health status in patients with COPD was not known in the medium and long-time intervention period intervention time. The studies on longtime intervention period Tai Chi and exercise intervention for COPD are expected to investigate the effects of Tai Chi on improving the health status of patients in the long-time intervention period intervention time.

HRQoL is an important indicator for evaluating the effectiveness of Tai Chi in patients with COPD. In this meta-analysis, five studies used the SGRQ $(32,33,36,39,42)$, and two studies used the CRQ $(8,40)$. Pooled analysis showed that there were a significant decrease of the SGRQ score and increase of the CRQ score of the Tai Chi group 


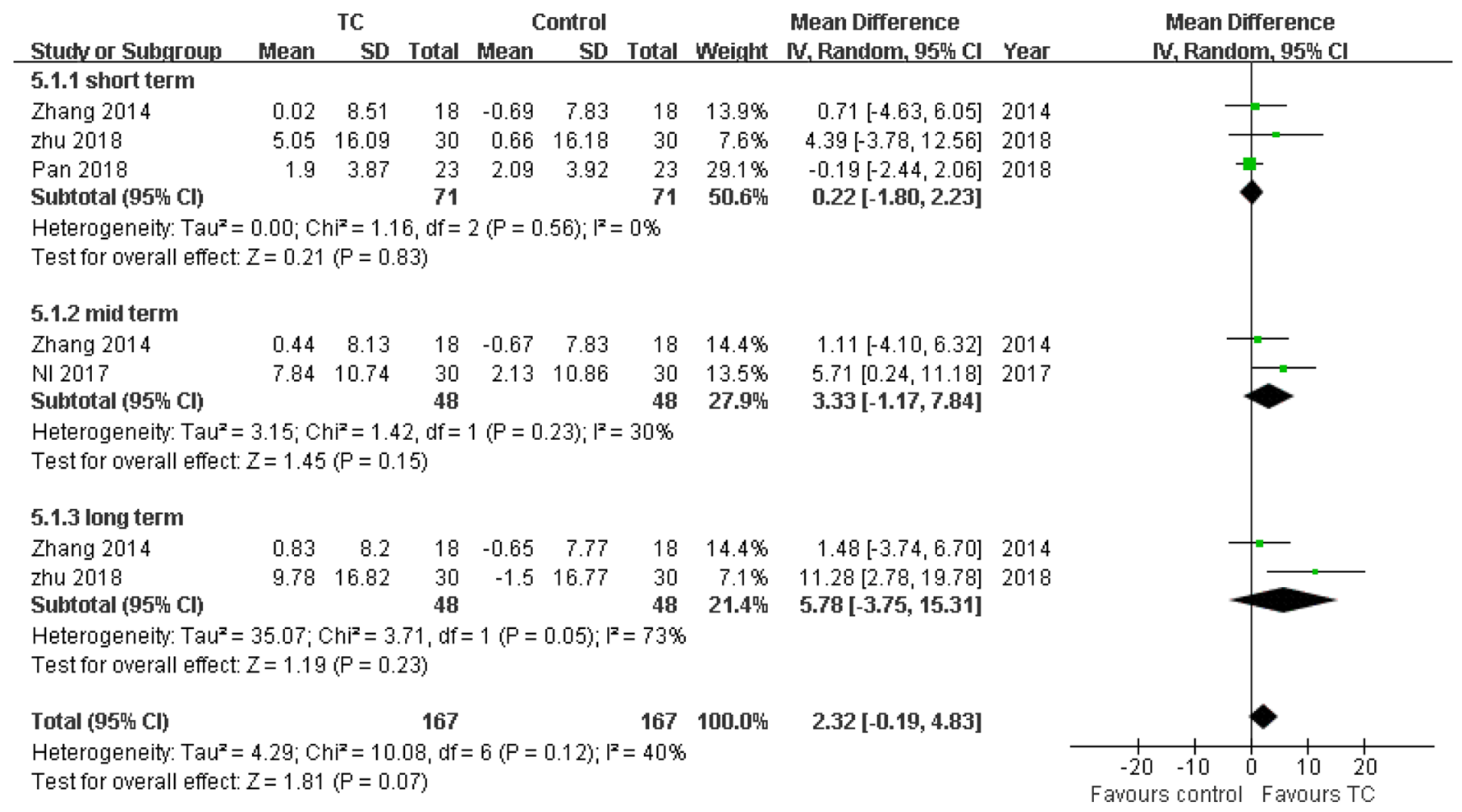

Figure 6 Meta-analysis of Tai on FEV1\%. Tai Chi group versus control group. Short time intervention period: $\leq 3$ months; medium time intervention period: 4-6 months; long time intervention period: $>6$ months. FEV1\%, FEV1 percent predicted normal values.

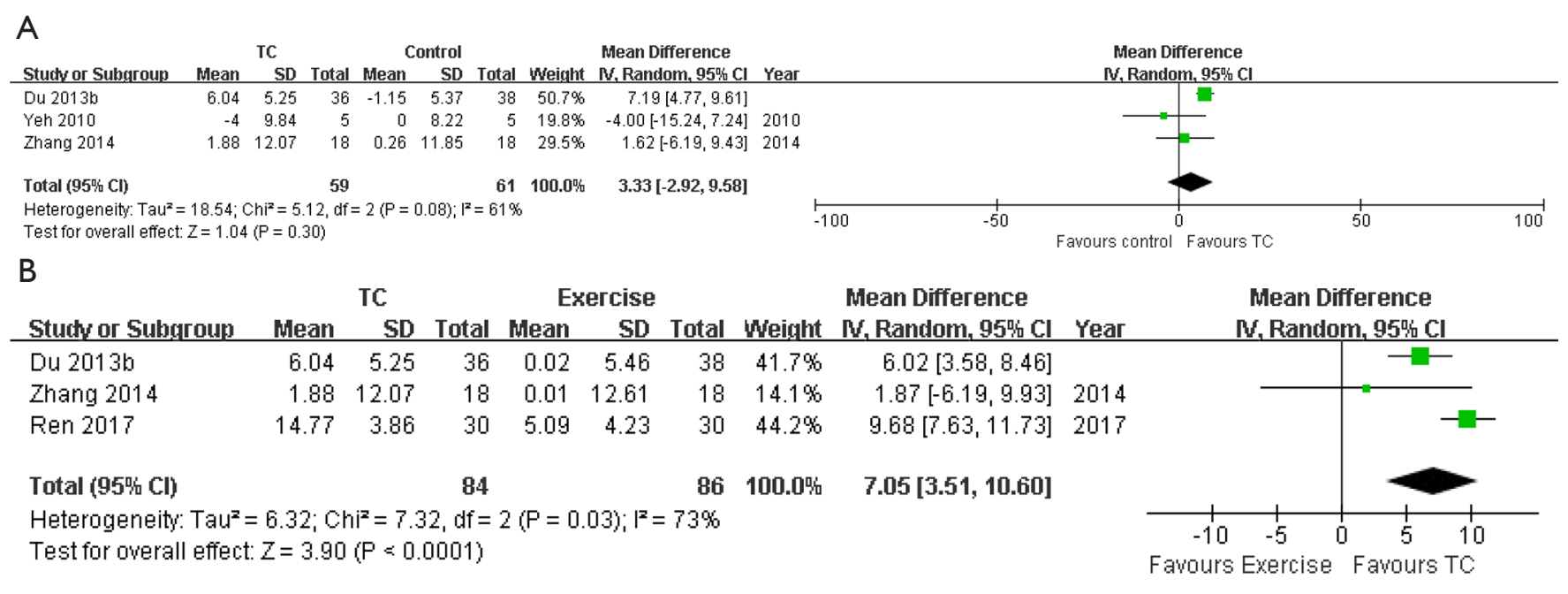

Figure 7 Meta-analysis of Tai on $\mathrm{FEV}_{1} / \mathrm{FVC}$ in short time intervention period. (A) Tai Chi group versus control group; (B) Tai Chi group versus exercise group. Short time intervention period: $\leq 3$ months; medium time intervention period: $4-6$ months; long time intervention period: $>6$ months. $\mathrm{FEV}_{1} / \mathrm{FVC}$, the ratio of $\mathrm{FEV}_{1}$ to $\mathrm{FVC}$. 
A

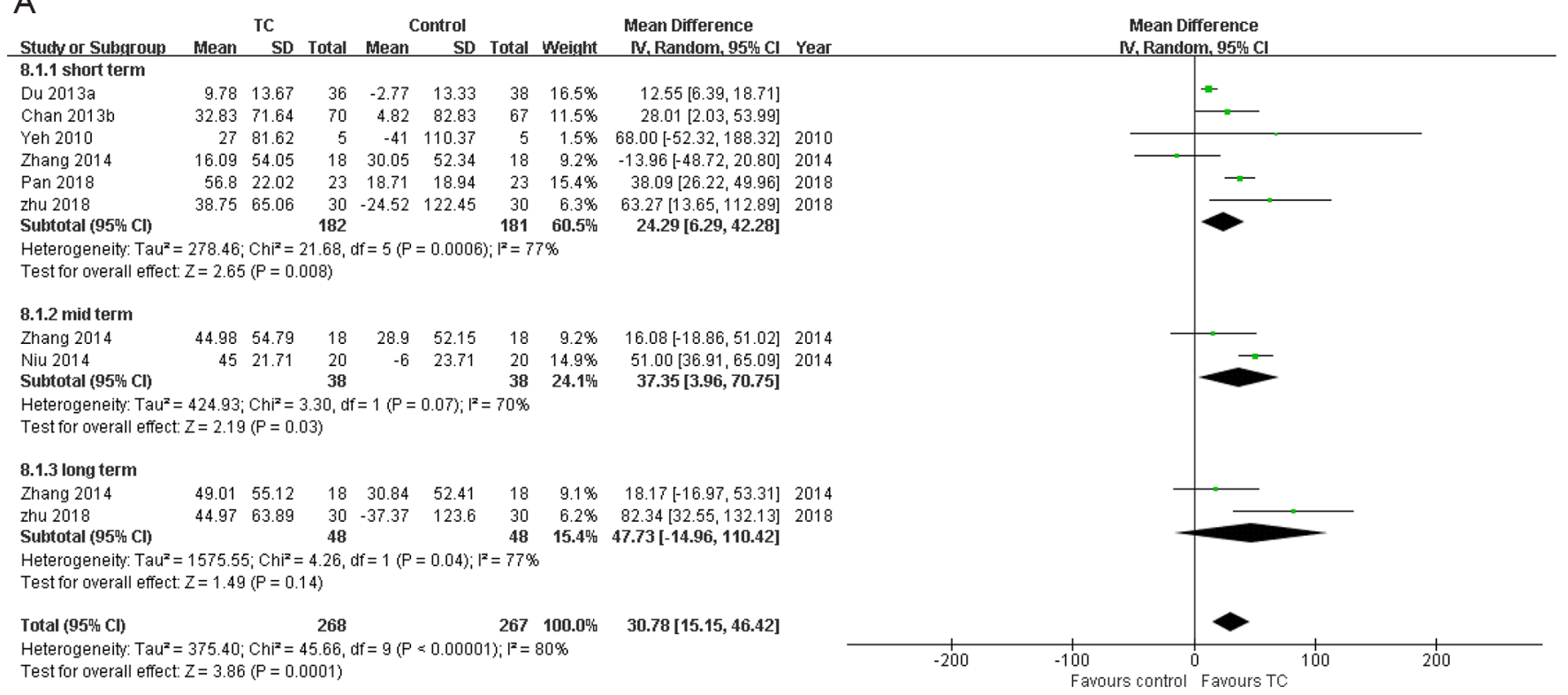

\section{B}

\begin{tabular}{|c|c|c|c|c|c|c|c|c|c|}
\hline \multirow[b]{2}{*}{ Studty or Subgroup } & \multicolumn{3}{|c|}{$\mathrm{TC}$} & \multicolumn{3}{|c|}{ Exercise } & \multicolumn{3}{|c|}{ Mlean Difference } \\
\hline & Mean & $\mathrm{SD}$ & Total & Mlean & SD & Total & Weight & W, Fixed, 95\% Cl & Year \\
\hline 9.1.1 short term & & & & & & & & & \\
\hline Du $2013 a$ & 9.78 & 13.67 & 36 & 2.67 & 12.84 & 38 & $72.2 \%$ & $7.11[1.06,13.16]$ & \\
\hline Chan 2013b & 32.83 & 71.64 & 70 & 5.4 & 87.2 & 69 & $3.8 \%$ & $27.43[0.88,53.98]$ & \\
\hline Zhang 2014 & 16.09 & 54.05 & 18 & 20.06 & 53.11 & 18 & $2.2 \%$ & $-3.97[-38.98,31.04]$ & 2014 \\
\hline $\lg 2014$ & 27.4 & 73.3 & 94 & 20.8 & 79.8 & 98 & $5.6 \%$ & $6.60[-15.06,28.26]$ & 2014 \\
\hline Ren 2017 & 54.2 & 90.45 & 30 & 22.89 & 73.59 & 30 & $1.5 \%$ & $31.31[-10.42,73.04]$ & 2017 \\
\hline Polkey 2018 & 19 & 22.02 & 60 & 18.71 & 73.95 & 60 & $6.9 \%$ & $0.29[-19.23,19.81]$ & 2018 \\
\hline Subtotal (95\% Cl) & & & 308 & & & 313 & 92.2 㗱 & $7.53[2.18,12.89]$ & \\
\hline
\end{tabular}

Heterogeneity: $\mathrm{Ch}^{2}=4.37, \mathrm{df}=5(\mathrm{P}=0.50) ; \mathrm{I}^{\mathrm{z}}=0 \%$

Test for owerall effect: $Z=2.76(\mathrm{P}=0.006)$

9.1.2 mid term

$\begin{array}{lrrrrrrrrr}\text { Zhang 2014 } & 44.98 & 54.79 & 18 & 41.13 & 53.07 & 18 & 2.1 \% & 3.85[-31.39,39.09] & 2014 \\ \text { Ng 2014 } & 28.1 & 76.24 & 94 & 14.9 & 76.93 & 98 & 5.6 \% & 13.20[-8.47,34.87] & 2014 \\ \text { Subtotal (95\% Cl) } & & & \mathbf{1 1 2} & & & \mathbf{1 1 6} & \mathbf{7 . 8 \%} & \mathbf{1 0 . 6 3}[-\mathbf{7 . 8 2 , 2 9 . 0 9 ]}\end{array}$

Heterogeneity: $\mathrm{Ch}^{2}=0.20, \mathrm{df}=1(\mathrm{P}=0.66) ; \mathrm{F}^{\mathbf{2}}=0 \%$

Test for owerall effect: $Z=1.13(\mathrm{P}=0.26)$
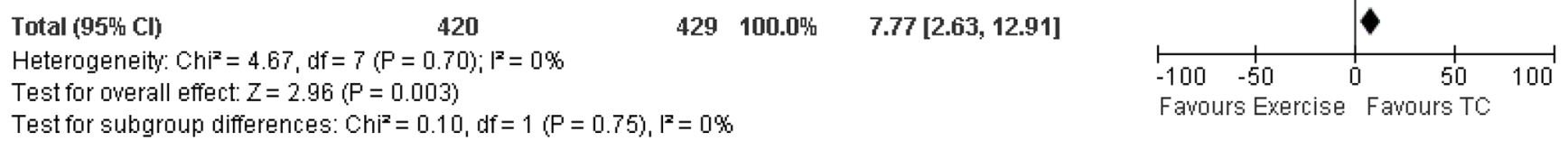

Figure 8 Meta-analysis of Tai on 6MWD. (A) Tai Chi group versus control group; (B) Tai Chi group versus exercise group. Short time intervention period: $\leq 3$ months; medium time intervention period: 4-6 months; long time intervention period: $>6$ months. $6 \mathrm{MWD}, 6$-minute walking distance. 
A

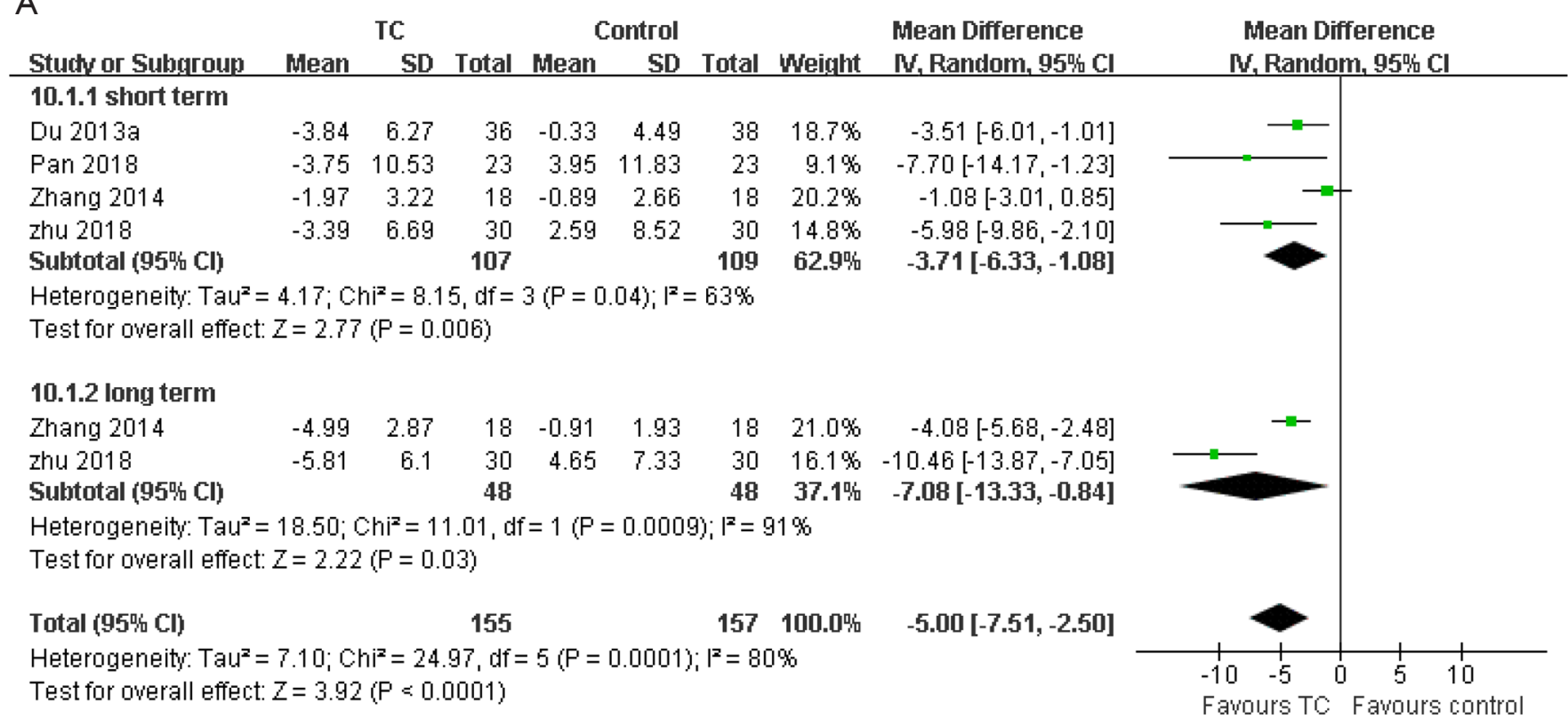

B

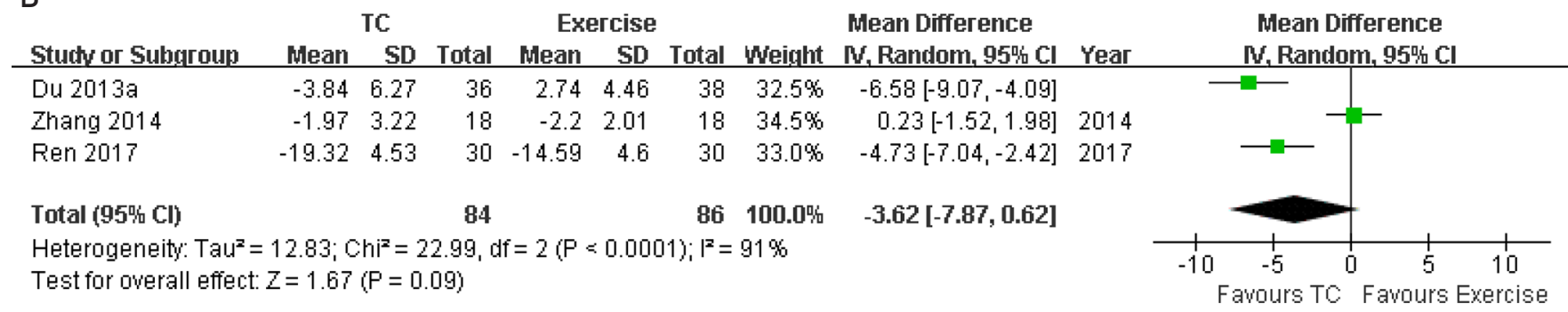

Figure 9 Meta-analysis of Tai on CAT. (A) Tai Chi group versus control group; (B) Tai Chi group versus exercise group in short time intervention period. Short time intervention period: $\leq 3$ months; medium time intervention period: $4-6$ months; long time intervention period: $>6$ months. CAT, COPD Assessment Test.

compared with the control group. The average changes in both scores were greater than their respective MCID (SGRQ: 4 points, CRQ: 0.5 points) (49,50). A number of studies have shown that Tai Chi can decrease the negative impact of COPD on patients' lives and improve their quality of life by improving the oxygen-absorbing capacity of patients with COPD, strengthening exercise endurance and lung function (34). The comparison of SGRQ scores between the Tai Chi group and the exercise group before and after the short-time intervention period intervention showed no statistical difference. In the original study, two of the four studies showed significant difference in SGRQ scores between the Tai Chi group and exercise group in short time intervention period $(39,42)$, one study showed the difference was not significant in the short time intervention period, but the long-time intervention period intervention was significantly different (32). It shows that the Tai Chi may represent an appropriate alternative or complement to standard rehabilitation programs in the improvement of HRQoL to patients with COPD. Moreover, more long-time intervention period studies are needed to confirm the conclusion.

In these studies investigating the impact of Tai Chi on the psychological status of patients with COPD, two studies used the HAD evaluation tool to access the difference between the Tai Chi group and the control group $(33,40)$, and one used the SAS and SDS as evaluation tools to access the difference between the Tai Chi group and the exercise group (34). Two studies using HAD showed that the improvement of anxiety and depression in the Tai Chi group was superior than the control group, and the difference was significant. The original studies comparing Tai Chi group 
A

\begin{tabular}{|c|c|c|c|c|c|c|c|c|c|}
\hline \multirow[b]{2}{*}{ Stucty or Subgroup } & \multicolumn{3}{|c|}{$\mathrm{TC}$} & \multicolumn{3}{|c|}{ Control } & \multicolumn{2}{|r|}{ Mean Difference } & \multirow{2}{*}{$\begin{array}{l}\text { Mean Difference } \\
\text { W, Random, 95㖡 Cl }\end{array}$} \\
\hline & hlean & $\mathrm{SD}$ & Total & hlean & SD & Total & Weight & W, Random, 95 唃 Cl & \\
\hline Chan 2013a & -0.92 & 16.6 & 70 & 4.04 & 17 & 67 & $35.6 \%$ & $-4.96[-10.59,0.67]$ & \\
\hline Du $2013 a$ & -11.26 & 14.42 & 36 & 4.84 & 19.24 & 38 & $27.8 \%$ & $-16.10[-23.82,-8.38]$ & \\
\hline Total (95㓍 Cl) & & & 129 & & & 128 & $100.0 \%$ & $-8.66[-14.60,-2.72]$ & \\
\hline \multicolumn{9}{|c|}{$\begin{array}{l}\text { Heterogeneity: Tau }{ }^{2}=17.59 ; \mathrm{Chi}^{2}=5.60, \mathrm{df}=2(\mathrm{P}=0.06) ; \mathrm{I}^{2}=64 \% \\
\text { Test for overall effect: } Z=2.86(\mathrm{P}=0.004)\end{array}$} & $\begin{array}{cccc}-20 & -10 & 0 & 10 \\
\text { Fawours } & \text { TC } & \text { Favours }\end{array}$ \\
\hline
\end{tabular}

B

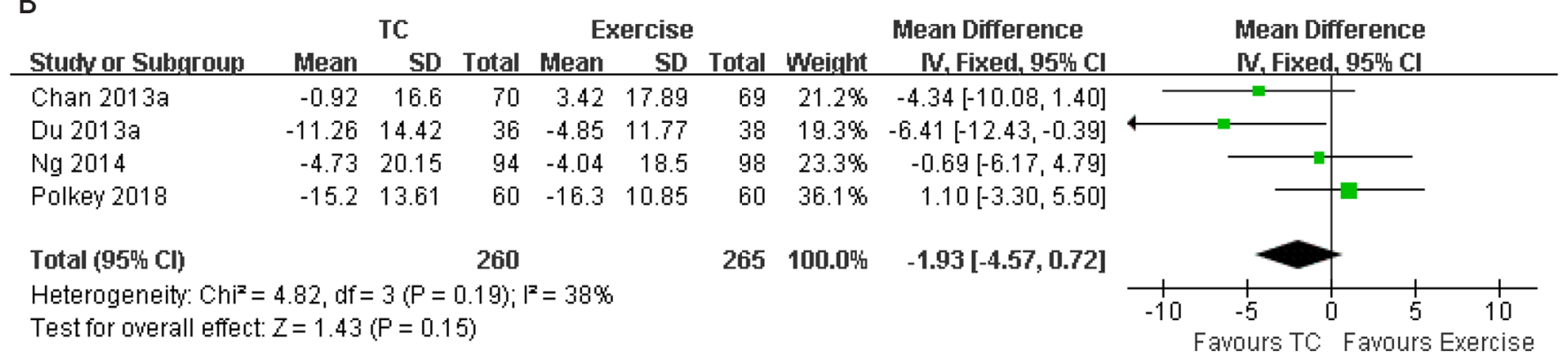

Figure 10 Meta-analysis of Tai on SGRQ in short time intervention period. (A) Tai Chi group versus control group; (B) Tai Chi group versus exercise group. Short time intervention period: $\leq 3$ months; medium time intervention period: $4-6$ months; long time intervention period: >6 months. SGRQ, St. George's Respiratory Questionnaire.

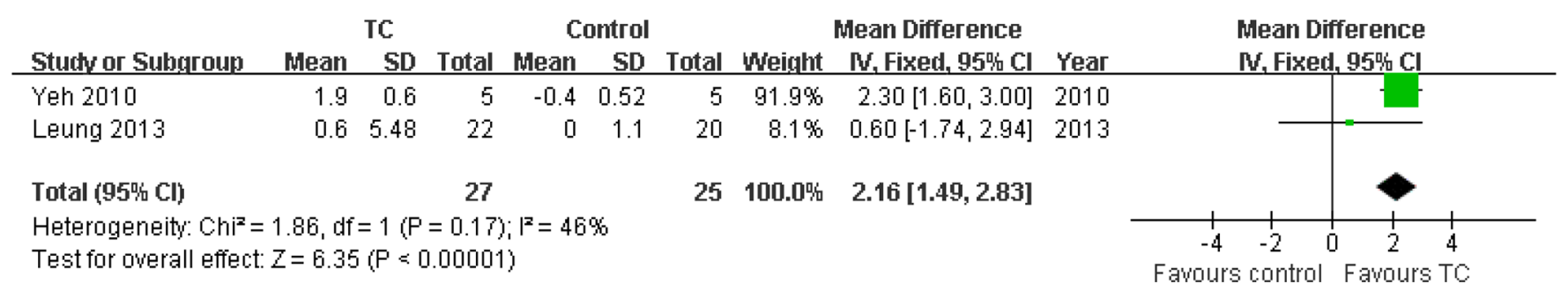

Figure 11 Meta-analysis of Tai on CRQ in short time intervention period. Tai Chi group versus control group. Short time intervention period: $\leq 3$ months; medium time intervention period: 4-6 months; long time intervention period: $>6$ months. CRQ, Chronic Respiratory Disease Questionnaire.

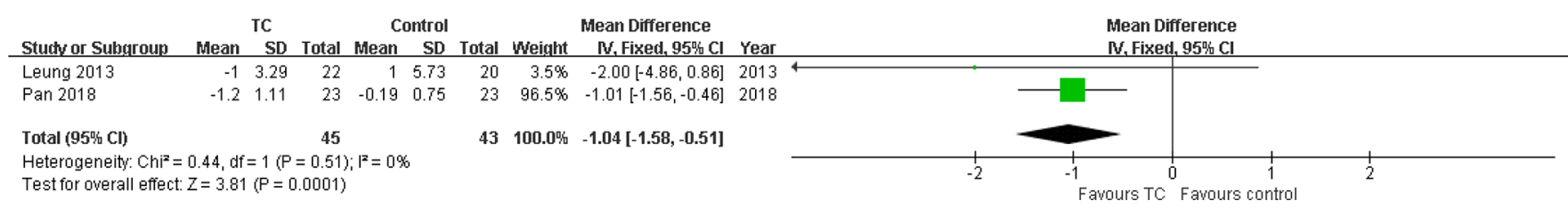

Figure 12 Meta-analysis of Tai on HAD (anxiety) in short time intervention period. Tai Chi group versus control group. Short time intervention period: $\leq 3$ months; medium time intervention period: 4-6 months; long time intervention period: $>6$ months. HAD, hospital anxiety and depression Scale. 


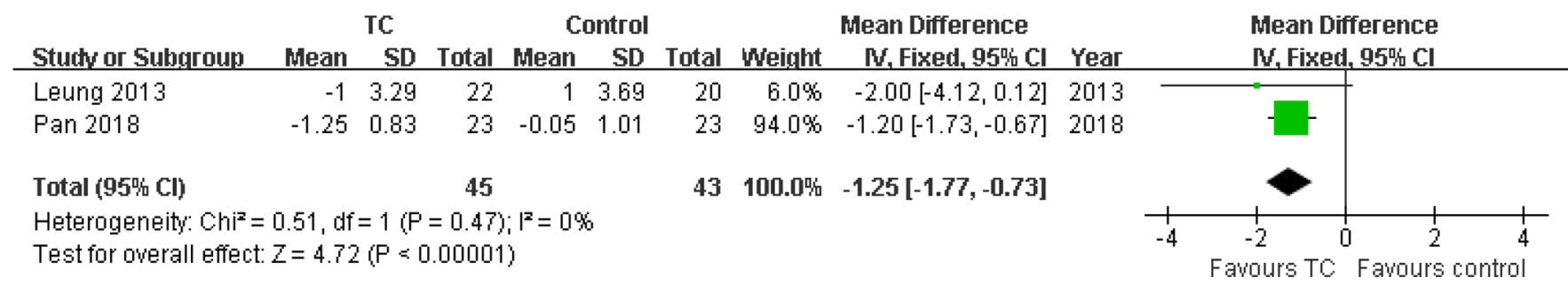

Figure 13 Meta-analysis of Tai on HAD (depression) in short time intervention period. Tai Chi group versus control group. Short time intervention period: $\leq 3$ months; medium time intervention period: 4-6 months; long time intervention period: $>6$ months. HAD, hospital anxiety and depression Scale.

Table 3 Summary of results

\begin{tabular}{|c|c|c|c|c|}
\hline Outcomes & No. of trials & No. of participants & Mean difference $(95 \% \mathrm{Cl})$ & Heterogeneity $\left(I^{2}\right)$ \\
\hline \multicolumn{5}{|l|}{ TC vs. control } \\
\hline Short term & 2 & 183 & $0.12(0.03,0.21) ; P=0.01$ & $0 \%$ \\
\hline \multicolumn{5}{|c|}{ TC vs. exercise } \\
\hline \multicolumn{5}{|l|}{$\mathrm{FEV}_{1}$} \\
\hline \multicolumn{5}{|l|}{ TC vs. control } \\
\hline Short term & 3 & 219 & $0.13(0.06,0.2) ; P=0.0002$ & $12 \%$ \\
\hline Mid term & 3 & 136 & 0.17 (0.04, 0.30); P=0.01 & $59 \%$ \\
\hline Mid term & 2 & 228 & $0.03(-0.09,0.14) ; P=0.62$ & $0 \%$ \\
\hline \multicolumn{5}{|l|}{$\mathrm{FEV}_{1} \%$} \\
\hline \multicolumn{5}{|l|}{ TC vs. control } \\
\hline Short term & 3 & 142 & $0.22(-1.80,2.23) ; P=0.83$ & $0 \%$ \\
\hline Mid term & 2 & 96 & $3.33(-1.17,7.84) ; P=0.15$ & $30 \%$ \\
\hline Long term & 2 & 96 & $5.78(-3.75,15.31) ; P=0.23$ & $73 \%$ \\
\hline \multicolumn{5}{|l|}{$\mathrm{FEV}_{1} / \mathrm{FVC}$} \\
\hline \multicolumn{5}{|l|}{ TC vs. control } \\
\hline
\end{tabular}

Table 3 (Continued) 
Table 3 (Continued)

\begin{tabular}{|c|c|c|c|c|}
\hline Outcomes & No. of trials & No. of participants & Mean difference (95\% Cl) & Heterogeneity $\left(I^{2}\right)$ \\
\hline \multicolumn{5}{|l|}{ TC vs. control } \\
\hline Short term & 6 & 363 & 24.29 (6.29, 42.28); $P=0.008$ & $77 \%$ \\
\hline Mid term & 2 & 76 & 37.35 (3.96, 70.75); $P=0.03$ & $70 \%$ \\
\hline \multicolumn{5}{|c|}{ TC vs. exercise } \\
\hline Short term & 6 & 621 & 7.53 (2.18, 12.89); P=0.006 & $0 \%$ \\
\hline Mid term & 2 & 228 & $10.63(-7.82,29.09) ; P=0.26$ & $0 \%$ \\
\hline \multicolumn{5}{|l|}{ CAT } \\
\hline Long term & 2 & 312 & $-7.08(-13.33,-0.84) ; P=0.03$ & $91 \%$ \\
\hline \multicolumn{5}{|c|}{ TC vs. exercise } \\
\hline Short term & 3 & 170 & $-3.62(-7.87,0.62) ; P=0.09$ & $91 \%$ \\
\hline \multicolumn{5}{|l|}{ SGRQ } \\
\hline \multicolumn{5}{|l|}{ TC vs. control } \\
\hline Short term & 3 & 257 & $-8.66(-14.60,-2.72) ; P=0.004$ & $64 \%$ \\
\hline \multicolumn{5}{|c|}{ TC vs. exercise } \\
\hline Short term & 4 & 525 & $-1.93(-4.57,0.72) ; P=0.15$ & $38 \%$ \\
\hline \multicolumn{5}{|l|}{ TC vs. control } \\
\hline \multicolumn{5}{|l|}{ HAD (anxiety) } \\
\hline Short term & 2 & 88 & $-1.04(-1.58,-0.51) ; P=0.0001$ & $0 \%$ \\
\hline \multicolumn{5}{|c|}{ HAD (depression) } \\
\hline \multicolumn{5}{|l|}{ TC vs. control } \\
\hline Short term & 2 & 88 & $-1.25(-1.77,-0.73) ; P<0.00001$ & $0 \%$ \\
\hline
\end{tabular}

TC, Tai Chi; FVC, forced volume capacity; $\mathrm{FEV}_{1} \%, \mathrm{FEV}_{1}$ percent predicted normal values; $\mathrm{FEV}_{1}$, forced expiratory volume in $1 \mathrm{~s}$; $\mathrm{FEV}_{1}$ / FVC, the ratio of FEV to FVC; 6MWD, six-minute walking distance; CAT, COPD Assessment Test; SGRQ, St. George's Respiratory Questionnaire; CRQ, Chronic Respiratory Disease Questionnaire; HAD, hospital anxiety and depression scale. 
with exercise group showed that the difference in anxiety and depression were not significant.

Pulmonary rehabilitation is a very effective program to improve the quality of life and exercise performance of patients with COPD. But once the course completed, the benefits will wane (32). Previous studies have shown that Tai Chi and PR play an equivalent role in improving the quality of life of patients, and Tai Chi can still improve the quality of life of patients after training. Therefore, Tai Chi can be used as an alternative to PR (32). Judging from the form of Tai Chi and exercise in PR, Tai Chi is a soft sport, which does not require high physical endurance to patients, and is suitable for the elderly. Our research suggested that it was unclear whether Tai Chi is better than traditional exercise in PR. Whatever, Tai Chi has clearly improved the physical and mental health of patients with COPD. Therefore, clinically, we can try to use Tai Chi instead of exercise in PR or a combination of the two for the elderly patients.

Our study has the following strengths compared with previous related studies. Firstly, according to the intervention method and the intervention time, we divided patients into Tai Chi group versus control group and Tai Chi group versus exercise group. We not only compared Tai Chi with no intervention, but also compared Tai Chi with exercise. In addition, we divided intervention time into short-time intervention period ( $<3$ months), mediumtime intervention period (4-6 months), and long-time intervention period ( $>6$ months), and we did the subgroup analysis which reduced the clinical heterogeneity and explored the length of the intervention to the influence on Tai Chi efficacy. Secondly, the mental status was analyzed. We not only paid close attention to the physiological status of patients with COPD, but also gave attention to psychological status of patients with COPD, and evaluated the influence of Tai Chi to patients with COPD comprehensively. Thirdly, pooled analysis included new study about the impact of Tai Chi on patients with COPD in the past two years, and all of these studies had higher scores and quality.

At the same time, our study has following limitations. Firstly, most of the included studies did not report blindness and allocation concealment, which lead to the negative impact of study quality evaluation. Secondly, most of the interventions included in these studies were short-time intervention period and medium-time intervention period. There were few studies on long-time intervention period interventions, which led to the failure of many outcome measures to compare the effects of different intervention times on Tai Chi. It is expected that higher quality articles to be carried out in the future, which will provide more high-quality evidence to support for the effects of Tai Chi intervention. In addition, we expected, in the future, studies could explore the length of intervention, the frequency and intensity of intervention, and form a set of optimal Tai Chi patterns in line with patients with COPD to maximize the advantages of Tai Chi in improving their general condition.

\section{Conclusions}

A program of Tai Chi lasting at least eight weeks has been shown to improve lung function, exercise capacity, health status, quality of life and mental status of patients with COPD. Whether the efficacy of Tai Chi is better than pulmonary rehabilitation exercise is not clear. Future studies with large sample size trails comparing Tai Chi and pulmonary rehabilitation exercises are needed for recommending Tai Chi as an alternative or a complement to standard pulmonary rehabilitation exercise programs.

\section{Acknowledgments}

Funding: This work was supported by grants from The National Key Research and Development Program of China (No. 2018YFC1313600) and the National Natural Science Foundation of China (No. 81570081, 81770083).

\section{Footnote}

Conflicts of Interest: The authors have no conflicts of interest to declare.

Ethical Statement: The authors are accountable for all aspects of the work in ensuring that questions related to the accuracy or integrity of any part of the work are appropriately investigated and resolved.

Open Access Statement: This is an Open Access article distributed in accordance with the Creative Commons Attribution-NonCommercial-NoDerivs 4.0 International License (CC BY-NC-ND 4.0), which permits the noncommercial replication and distribution of the article with the strict proviso that no changes or edits are made and the original work is properly cited (including links to both the formal publication through the relevant DOI and the license). See: https://creativecommons.org/licenses/by-nc-nd/4.0/. 


\section{References}

1. Wieland LS, Santesso N. A summary of a Cochrane Review: Tai chi for chronic obstructive pulmonary disease (COPD). Eur J Integr Med 2016;8:894-5.

2. WHO. Chronic obstructive pulmonary disease (COPD); 2018.

3. Qaseem A, Wilt TJ, Weinberger SE, et al. Diagnosis and Management of Stable Chronic Obstructive Pulmonary Disease: A Clinical Practice Guideline Update from the American College of Physicians, American College of Chest Physicians, American Thoracic Society, and European Respiratory Society. Ann Intern Med 2011;155:179-91.

4. Wu W, Liu X, Wang L, et al. Effects of Tai Chi on exercise capacity and health-related quality of life in patients with chronic obstructive pulmonary disease: a systematic review and meta-analysis. Int J Chron Obstruct Pulmon Dis 2014;9:1253-63.

5. Ngai SPC, Jones AYM, Tam WWS. Tai Chi for chronic obstructive pulmonary disease (COPD). Cochrane Database Syst Rev 2016;(6):CD009953.

6. Solloway MR, Taylor SL, Shekelle PG, et al. An evidence map of the effect of Tai Chi on health outcomes. Syst Rev 2016;5:126.

7. Yao YP. Effect of Tai Chi Chuan on chronic obstructive pulmonary disease. Chin J Rehabil Med 2004;10:439-40.

8. Yeh GY, Roberts DH, Wayne PM, et al. Tai chi exercise for patients with chronic obstructive pulmonary disease: a pilot study. Respir Care 2010;55:1475-82.

9. Zhang LH, Wu JJ, Wang ZC. Effects of 24-form Tai Chi with respiratory rehabilitation training on chronic obstructive pulmonary disease. Acta Univ Tradit Med Sin Pharm Shanghai 2012;2:53-6.

10. Zhu S, Shi K, Yan J, et al. A modified 6-form Tai Chi for patients with COPD. Complement Ther Med 2018;39:36-42.

11. Guo J, Chen B, Lu Y, et al. Tai Chi for improving cardiopulmonary function and quality of life in patients with chronic obstructive pulmonary disease: a systematic review and meta-analysis. Clin Rehabil 2016;30:750-64.

12. Moher D, Liberati A, Tetzlaff J, et al. Preferred reporting items for systematic reviews and meta-analyses: the PRISMA statement. BMJ 2009;339:b2535.

13. Hu Y, Li XL, Hao YF, et al. Evidence-based nursing. Beijing: People's Medical Publishing House, 2012:120-1.

14. Cui CM, Xing J, Yang YP, et al. Effect of Taijiquan exercise on pulmonary function in patients with chronic obstructive pulmonary disease in middle-aged stable period. Chin J Women Child Health Res 2016.

15. Gu G, Zhou YM, Wang DL, et al. Effects of shadow boxing training on exercise endurance and quality of life of patients with chronic obstructive pulmonary disease. Zhonghua Yi Xue Za Zhi 2012;92:952-5.

16. Polkey MI, Qiu Z, Zhou L, et al. Comparison of the adjunctive effects of Tai Chi and pulmonary rehabilitation on the effect of indacaterol in treatment naive patients with COPD. Am J Resp Crit Care 2017;195:A2852.

17. Dong L, Ma ZJ. Effect of Taijiquan Exercise on Rehabilitation of Patients with COPD. Cont Sp Tech 2015;5:17-8.

18. Zhang SS. Effect of Health Preserving Taijiquan on Rehabilitation of Chronic Obstructive Pulmonary Disease Patients. World Lat Med Inf 2017;17:4-5.

19. Yeh GY, Wayne PM, Litrownik D, et al. Tai chi mindbody exercise in patients with COPD: study protocol for a randomized controlled trial. Trials 2014;15:337.

20. Fu J, Min J, Yu P, et al. Study design for a randomised controlled trial to explore the modality and mechanism of Tai Chi in the pulmonary rehabilitation of chronic obstructive pulmonary disease. BMJ Open 2016;6:e011297.

21. Jones A. Sun-style T'ai Chi improves walking endurance and health-related quality of life in people with COPD. J Physiother 2013;59:273.

22. Lee MS, Chan AWK, Lee A, et al. Tai chi qigong may improve health-related QoL in chronic obstructive pulmonary disease patients. Focus on Alternative \& Complementary Therapies 2011;16:68-9.

23. Moy ML, Wayne PM, Litrownik D, et al. Long-term Exercise After Pulmonary Rehabilitation (LEAP): Design and rationale of a randomized controlled trial of Tai Chi. Contemp Clin Trials 2015;45:458-67.

24. Anonymous. Can Tai Chi improve respiratory function in patients with COPD? Perspect Public Heal 2018;138:183.

25. Yeh GY. Should you recommend Tai Chi for your patients with COPD? Journal of Respiratory Diseases 2005;26:405-6.

26. Kiatboonsri S, Amomputtisathaporn N, Siriket S, et al. Tai Chi Qigong exercise training in COPD. Chest 2007;132S:535S.

27. Leung RWM, Mckeough ZJ, Peters MJ, et al. The acceptability of Tai Chi in Caucasian people with COPD. Respirology 2013;184:179.

28. Leung R, McKeough Z, Peters M, et al. The Tai Chi Experience in people with COPD. Respirology 2012;171:59. 
29. Li Q, Fang WH, Lin C. The effect of taijiquan combined with respiratory exercise training on rehabilitation of patients with stable chronic obstructive pulmonary disease. Chin J Rehabil Med 2012;27:825-8.

30. Li S, Li A, Sun J, et al. Effects of Tai Chi Quan rehabilitation in patients with chronic obstructive pulmonary disease. Journal of Binzhou Medical University 2016;284:275-7.

31. Deng ZX, Chen H, Xu ZB, et al. The role of Taijiquan in pulmonary rehabilitation of chronic obstructive pulmonary disease in stable phase. J Anhui Heal Voc Tech Coll 2016;15:18-9.

32. Polkey MI, Qiu Z, Zhou L, et al. Tai Chi and Pulmonary Rehabilitation Compared for Treatment-Naive Patients With COPD A Randomized Controlled Trial. Chest 2018;153:1116-24.

33. Pan Y, Wang ZX, Min J, et al. The effect of 24 simplified Taichi on pulmonary rehabilitation in patients with stable chronic obstructive pulmonary disease. Chin J Rehabil Med 2018;33:681-6.

34. Ren ZF, Zhang X, Hou GH, et al. Effects of Tai Chi Zen on exercise ability and quality of life in chronic obstructive pulmonary disease. Chin J Trad Chin Med Pha 2017;32:3507-10.

35. Ni L. Therapeutic effect of yoga and Taiji on COPD remission. World Lat Med Inf 2017;17:182-4.

36. Ng L, Chiang LK, Tang R, et al. Effectiveness of incorporating Tai Chi in a pulmonary rehabilitation program for Chronic Obstructive Pulmonary Disease (COPD) in primary care-A pilot randomized controlled trial. Eur J Integr Med 2014;6:248-58.

37. Niu R, He R, Luo B, et al. The Effect of Tai Chi on Chronic Obstructive Pulmonary Disease: A Pilot Randomised Study of Lung Function, Exercise Capacity and Diaphragm Strength. Heart Lung Circ 2014;23:347-52.

38. Zhang XC, Cai YL, Zhang W, et al. Effects of 24form Tai Chi combined with respiratory training and western medicine on stable COPD. J Trad Chin Med 2014;(22):1937-41.

39. Chan AW, Lee A, Lee DT, et al. Evaluation of the sustaining effects of Tai Chi Qigong in the sixth month in promoting psychosocial health in COPD patients: a singleblind, randomized controlled trial. ScientificWorldJournal 2013;2013:425082.
40. Leung RWM, McKeough ZJ, Peters MJ, et al. Short-form Sun-style t'ai chi as an exercise training modality in people with COPD. Eur Respir J 2013;41:1051-7.

41. Chan AWK, Lee A, Lee DTF, et al. The sustaining effects of Tai chi Qigong on physiological health for COPD patients: A randomized controlled trial. Complement Ther Med 2013;21:585-94.

42. Du ST, Xing B, Wang CX, et al. The effects of taijiquan practice on the BODE and SGRQ of COPD patients. Chin J Sports Med 2013;32:403-7.

43. Du ST, Ding LM, Wang CX, et al. The effects taijiquan practice on exercise endurance and lung function of patients with chronic obstructive pulmonary disease. Chin J Rehabil Med 2013;28:374-6.

44. Chan AW, Lee A, Suen LK, et al. Tai chi Qigong improves lung functions and activity tolerance in COPD clients: A single blind, randomized controlled trial. Complement Ther Med 2011;19:3-11.

45. Zhou Y, Wu L, Wang DX. The rehabilitative effects of Taijiquan training with east medical on middle-elderly COPD patients. J Jinlin I Phys Educ 2009;25:54-5.

46. Mannino DM, Watt G, Hole D, et al. The natural history of chronic obstructive pulmonary disease. Eur Respir J 2006;27:627.

47. Holland AE, Nici L. The return of the minimum clinically important difference for 6-minute-walk distance in chronic obstructive pulmonary disease. Am J Respir Crit Care Med 2013;187:335-6.

48. Kon SSC, Canavan JL, Jones SE, et al. Minimum clinically important difference for the COPD Assessment Test: a prospective analysis. The Lancet Respiratory Medicine 2014;2:195-203.

49. Jones PW. St. George's Respiratory Questionnaire: MCID. COPD 2005;2:75-9.

50. Schünemann HJ, Puhan M, Goldstein R, et al. Measurement properties and interpretability of the chronic respiratory disease questionnaire (CRQ). COPD 2005;2:81-9.

Cite this article as: Guo C, Xiang G, Xie L, Liu Z, Zhang X, Wu Q, Li S, Wu Y. Effects of Tai Chi training on the physical and mental health status in patients with chronic obstructive pulmonary disease: a systematic review and meta-analysis. J Thorac Dis 2020;12(3):504-521. doi: 10.21037/jtd.2020.01.03 
Table S1 PRISMA 2009 checklist word Section/topic \# Checklist item

Title

Title

2 Provide a structured summary including, as applicable: background; objectives; data sources; study eligibility criteria, participants, and interventions; study appraisal and synthesis methods; results; limitations; conclusions

Introduction

Rationale and implications of key findings; systematic review registration number

Objectives

Methods

Protocol and registration

Eligibility criteria

Information sources

Search

Study selection

Data collection process

Data items

Risk of bias in individual studies

Summary measures

Synthesis of results

Risk of bias across studies

Additional analyses

Results

Study selection

Study characteristics

Risk of bias within studies

Results of individual studies

Synthesis of results

Risk of bias across studies

Describe the rationale for the review in the context of what is already known.

4 Provide an explicit statement of questions being addressed with reference to participants, interventions, comparisons, outcomes, and study design (PICOS).

5 Indicate if a review protocol exists, if and where it can be accessed (e.g., Web address), and, if available, provide registration information including registration number.

6 Specify study characteristics (e.g., PICOS, length of follow-up) and report characteristics (e.g., years considered, language, publication status) used as criteria for eligibility, giving rationale.

7 Describe all information sources (e.g. databases with dates of coverage, contact with study authors to identify additional studies) in the search and date last searched.

8 Present full electronic search strategy for at least one database, including any limits used, such that it could be repeated.

9 State the process for selecting studies (i.e., screening, eligibility, included in systematic review, and, if applicable, included in the meta-analysis).

10 Describe method of data extraction from reports (e.g., piloted forms, independently, in duplicate) and any processes for obtaining and confirming data from investigators.

11 List and define all variables for which data were sought (e.g., PICOS, funding sources) and any assumptions and simplifications made.

12 Describe methods used for assessing risk of bias of individual studies (including specification of whether this was done at the study or outcome level), and how this information is to be used in any data synthesis.

13 State the principal summary measures (e.g., risk ratio, difference in means).

14 Describe the methods of handling data and combining results of studies, if done, including measures of consistency (e.g., $1^{2}$ ) for each meta-analysis.

15 Specify any assessment of risk of bias that may affect the cumulative evidence (e.g., publication bias, selective reporting within studies).

16 Describe methods of additional analyses (e.g., sensitivity or subgroup analyses, meta-regression), if done, indicating which were pre-specified.

17 Give numbers of studies screened, assessed for eligibility, and included in the review, with reasons for exclusions at each stage, ideally with a flow diagram.

18 For each study, present characteristics for which data were extracted (e.g., study size, PICOS, follow-up period) and provide the citations.

19 Present data on risk of bias of each study and, if available, any outcome level assessment (see item 12).

For all outcomes considered (benefits or harms), present, for each study: (a) simple summary data for each intervention group (b) effect estimates and confidence intervals, ideally with a forest plot.

21 Present results of each meta-analysis done, including confidence intervals and measures of consistency.

22 Present results of any assessment of risk of bias across studies (see Item 15).

23 Give results of additional analyses, if done [e.g., sensitivity or subgroup analyses, meta-regression (see Item 16)].

Discussion

Summary of evidence

Limitations

24 Summarize the main findings including the strength of evidence for each main outcome; consider their relevance to key groups (e.g., healthcare providers, users, and policy makers)

25 Discuss limitations at study and outcome level (e.g., risk of bias), and at review-level (e.g., incomplete retrieval of identified research, reporting bias).

$9-11$

$11-13$

$10-23$

Conclusions

26 Provide a general interpretation of the results in the context of other evidence, and implications for future research. 


\section{Supplementary file 1}

\section{Research strategy}

Appendix: search strategies

PubMed

\#1 tai ji[MeSH Terms]

\#2 tai ji[Title/Abstract]) OR tai ji quan[Title/Abstract]) OR

tai chi[Title/Abstract]) OR tai chi chuan[Title/Abstract])

OR t'ai chi [Title/Abstract]

\#3 \#1 or \#2

\#4 pulmonary disease, chronic obstructive[MeSH Terms]

\#5 copd [Title/Abstract] OR chronic obstructive pulmonary

disease [Title/Abstract] OR chronic obstructive airway

disease [Title/Abstract]OR chronic obstructive lung

disease[Title/Abstract]

\#6 \#4 or \#5

$\# 7$ \#3 and \#6

Cochrane

\#1 MeSH descriptor: [Tai Ji] explode all trees

\#2 tai ji[Title/Abstract]) OR tai ji quan[Title/Abstract]) OR

tai chi[Title/Abstract]) OR tai chi chuan[Title/Abstract])

OR t'ai chi [Title/Abstract]

\#3 \#1 or \#2

\#4 MeSH descriptor: [Pulmonary Disease, Chronic Obstructive] explode all trees

\#5 copd [Title/Abstract] OR chronic obstructive pulmonary disease [Title/Abstract] OR chronic obstructive airway disease [Title/Abstract]OR chronic obstructive lung disease[Title/Abstract]

\#6 \#4 or \#5

$\# 7 \# 3$ and \#6

\section{JBI}

\#1 (tai ji or tai ji quan or tai chi or tai chi chuan or t'ai chi). m_titl.

\#2 (tai ji or tai ji quan or tai chi or tai chi chuan or t'ai chi). $\mathrm{mp}$. [mp=text, heading word, subject area node, title]

\#3 \#1 or \#2

\#4 (copd or chronic obstructive pulmonary disease or chronic obstructive airway disease or chronic obstructive lung disease).mp. [mp=text, heading word, subject area node, title]
\#5 (copd or chronic obstructive pulmonary disease or chronic obstructive airway disease or chronic obstructive lung disease).m_titl.

\#6 \#4 or \#5

$\# 7 \# 3$ and \#6

\section{CINAHL}

\#1 tai ji[Title/Abstract]) OR tai ji quan[Title/Abstract]) OR tai chi[Title/Abstract]) OR tai chi chuan[Title/Abstract]) OR t'ai chi [Title/Abstract]

\#2 copd [Title/Abstract] OR chronic obstructive pulmonary disease [Title/Abstract] OR chronic obstructive airway disease [Title/Abstract]OR chronic obstructive lung disease [Title/Abstract]

\#3 \#1 and \#2

Web of science

\#1 (tai ji or tai ji quan or tai chi or tai chi chuan or t'ai chi)Databases $=$ WOS, BIOSIS, CCC, DIIDW, KJD, MEDLINE, RSCI, SCIELO

\#2 (copd or chronic obstructive pulmonary disease or chronic obstructive airway disease or chronic obstructive lung disease)

Databases $=$ WOS, BIOSIS, CCC, DIIDW, KJD, MEDLINE, RSCI, SCIELO

$\# 3 \# 1$ and \#2

Embase

\#1 "copd":ab,ti OR "chronic obstructive airway disease":ab,ti OR "chronic obstructive lung disease":ab,ti OR "chronic obstructive pulmonary disease":ab,ti

\#2 "pulmonary disease, chronic obstructive"/exp

\#3 \#1 or \#2

\#4 "tai ji”/exp

\#5 “tai ji”:ab,ti OR “tai ji quan":ab,ti OR "tai chi”:ab,ti OR "tai chi chuan":ab,ti OR "t'ai chi”: ab,ti

\#6 \#4 or \#5

$\# 7$ \#6 and \#3

CBM

\#1 "tai ji quan"

\#2 "tai ji quan"[Title] OR "tai ji”[Title]

\#3 “tai ji quan”[Abstract] OR "tai ji”[Abstract] 
\#4 “tai ji quan "[ Keyword] OR "tai ji"[ Keyword]

$\# 5 \# 1$ or \#2 or \#3 or \#4

\#6 “COPD”[Title]) OR “chronic obstructive pulmonary disease"[ Title]) OR "copd"[Title])

\#7 "COPD" [Abstract]) OR "chronic obstructive pulmonary disease "[Abstract ])OR " copd "[ Abstract ])

\#8 "COPD"[ Keyword ]) OR " chronic obstructive pulmonary disease "[ Keyword ])OR "copd"[ Keyword])

\#9 \#6 or \#7 or \#8

$\# 10$ \#5 and \#9

\section{Wanfang}

\#1 Subject: "tai ji quan" or "tai ji"

\#2 Subject: "COPD" or "chronic obstructive pulmonary disease" or "copd"

\#3 \#1 and \#2

\section{$\mathrm{CNKI}$}

\#1 SU="tai ji” OR SU="tai ji quan"

\#2 SU="COPD” OR SU=“chronic obstructive pulmonary disease" OR SU="copd"

\#3 \#1 and \#2 